\title{
On Stable Local Bases for Bivariate Polynomial Spline Spaces
}

\author{
Oleg Davydov ${ }^{1)}$ and Larry L. Schumaker ${ }^{2)}$
}

\begin{abstract}
Stable locally supported bases are constructed for the spaces $\mathcal{S}_{d}^{r}(\triangle)$ of polynomial splines of degree $d \geq 3 r+2$ and smoothness $r$ defined on triangulations $\triangle$, as well as for various superspline subspaces. In addition, we show that for $r \geq 1$, it is impossible to construct bases which are simultaneously stable and locally linearly independent.
\end{abstract}

\section{$\S 1$. Introduction}

This paper deals with the classical space of polynomial splines

$$
\mathcal{S}_{d}^{r}(\triangle):=\left\{s \in C^{r}(\Omega):\left.s\right|_{T} \in \mathcal{P}_{d} \text { for all triangles } T \in \triangle\right\}
$$

where $\mathcal{P}_{d}$ is the space of polynomials of degree $d$, and $\triangle$ is a regular triangulation of a polygonal set $\Omega$. We also discuss superspline subspaces of the form

$$
\mathcal{S}_{d}^{r, \rho}(\triangle):=\left\{s \in \mathcal{S}_{d}^{r}(\triangle): s \in C^{\rho_{v}}(v) \text { for all } v \in \mathcal{V}\right\}
$$

with $\rho:=\left\{\rho_{v}\right\}_{v \in \mathcal{V}}$, where $\rho_{v}$ are given integers such that $r \leq \rho_{v} \leq d$, and $\mathcal{V}$ is the set of all vertices of $\triangle$.

Our aim is to describe algorithms for constructing locally supported bases $\left\{B_{i}\right\}_{i \in \mathcal{I}}$ for these spaces which are stable in the following sense:

$$
K_{1}\|c\|_{\infty} \leq\left\|\sum_{i \in \mathcal{I}} c_{i} B_{i}\right\|_{\infty} \leq K_{2}\|c\|_{\infty}
$$

for all choices of the coefficient vector $c=\left(c_{i}\right)_{i \in \mathcal{I}}$. We are interested in a construction for which (1.2) holds with constants $K_{1}$ and $K_{2}$ which depend only on $d$ and the smallest angle $\theta_{\triangle}$ in the triangulation, and not on the number of triangles or any other property of $\triangle$. Stable bases are of critical importance for both theoretical and practical purposes.

1) Mathematical Institute, Justus Liebig University, D-35392 Giessen, Germany, oleg.davydov@math.uni-giessen.de

2) Department of Mathematics, Vanderbilt University, Nashville, TN 37240, s@mars.cas.vanderbilt.edu. Supported by the National Science Foundation under grant DMS-9803340 and by the Army Research Office under grant DAAD-19-99-1-0160. 
To date, algorithms which produce stable local bases have been described only for certain very special spline spaces, see Remarks 13.2-13.5. In this paper we give algorithms to construct locally supported stable bases for general superspline spaces $\mathcal{S}_{d}^{r, \rho}(\triangle)$ with $d \geq 3 r+2$. Such bases for the full spline spaces $\mathcal{S}_{d}^{r}(\triangle$ ) (which correspond to the choice $\rho_{v}=r$ for all $v \in \mathcal{V}$ ) are of special importance due to the fact that these spaces are nested for nested triangulations, while most superspline spaces are not, see Remark 13.6.

The paper is organized as follows. In Sects. $2-4$ we review the BernsteinBézier framework for dealing with bivariate splines and the minimal determining set approach to constructing bases for spline spaces. In Sect. 5 we describe how to construct corresponding dual basis splines. Sect. 6 contains a special construction of minimal determining sets for superspline spaces defined on near-singular cells. Sects. 7 and 8 deal with near-degenerate edges and near-singular vertices. In Sect. 9 we construct stable local bases for the superspline space $\mathcal{S}_{d}^{r, \mu}(\triangle)$ defined by choosing $\rho_{v}=\mu$ for all $v \in \mathcal{V}$, where

$$
\mu:=r+\left\lfloor\frac{r+1}{2}\right\rfloor
$$

In Sect. 10 we consider superspline spaces on arbitrary cells. The main result for general superspline spaces $\mathcal{S}_{d}^{r, \rho}(\triangle)$ is established in Sect. 11, and the connection between stability and local linear independence is explored in Sect. 12. We conclude the paper with several remarks in Sect. 13.

\section{$\S 2$. The B-form of polynomials}

In this section we briefly recall some well-known notation. Let $T:=\langle u, v, w\rangle$ be a triangle in the plane. Throughout the paper, whenever we write a triangle $T$ in terms of its vertices, we assume that $u, v, w$ appear in counterclockwise order.

Given an integer $d$, we write $\left\{B_{i j k}^{d}\right\}_{i+j+k=d}$ for the usual Bernstein polynomials of degree $d$ associated with $T$. Then it is well known (cf. [12]) that every polynomial $p \in \mathcal{P}_{d}$ can be written uniquely in the form

$$
p=\sum_{i+j+k=d} c_{i j k} B_{i j k}^{d}
$$

The expansion (2.1) is called the Bernstein Bézier (B-) representation of $p$, and the $c_{i j k}$ are called the B-coefficients. It is known that the $B_{i j k}^{d}$ form a stable basis for $\mathcal{P}_{d}$, cf. Lemma 4.1 of [25].

We follow the standard convention of associating the B-coefficient $c_{i j k}$ with the domain point

$$
\xi_{i j k}^{T}:=(i u+j v+k w) / d .
$$

Thus, the coefficients of $p$ can be indexed with the set

$$
\mathcal{D}_{d, T}:=\left\{\xi_{i j k}^{T}\right\}_{i+j+k=d}
$$




\section{$\S 3$. The B-form of splines}

Given a triangulation $\triangle$, it is well known that there is a one-one correspondence between the spline space $\mathcal{S}_{d}^{0}(\triangle)$ and the set of coefficients $\left\{c_{\xi}\right\}_{\xi \in \mathcal{D}_{d, \Delta}}$, where

$$
\mathcal{D}_{d, \triangle}:=\bigcup_{T \in \triangle} \mathcal{D}_{d, T}
$$

In particular, $\left\{c_{\xi}\right\}_{\xi \in T \cap \mathcal{D}_{d, \triangle}}$ are the B-coefficients of $\left.s\right|_{T}$.

This means that the set $\mathcal{D}_{d, \triangle}$ can be used to parametrize $\mathcal{S}_{d}^{0}(\triangle)$. It is common to write $\lambda_{\xi}$ for the linear functional which picks off the B-coefficient $c_{\xi}$ of $s \in \mathcal{S}_{d}^{0}(\triangle)$.

We recall some additional standard notation. Given $T=\langle u, v, w\rangle$, we define the distance of the domain point $\xi_{i j k}^{T}$ from the vertex $u$ to be $\operatorname{dist}\left(\xi_{i j k}^{T}, u\right):=d-i$, with similar definitions for the other two vertices. We define the distance of $\xi_{i j k}^{T}$ from the edge $\langle v, w\rangle$ to be $i$, with similar definitions for the other two edges.

Given a vertex $u$, the ring of radius $m$ around $u$ is the set $R_{m}(u):=\{\eta$ : $\operatorname{dist}(\eta, u)=m\}$. The disk of radius $m$ around $u$ is $D_{m}(u):=\{\eta: \operatorname{dist}(\eta, u) \leq m\}$. We also define the $\operatorname{arc} a_{m, e}^{r}(u)$ around $u$ associated with an edge $e:=\langle u, w\rangle$ to be the set of domain points in the ring $R_{m}(u)$ whose distance to $\langle u, w\rangle$ is at most $r$.

\section{$\S 4$. Minimal determining sets}

Suppose $\mathcal{S}$ is a linear subspace of $\mathcal{S}_{d}^{0}(\triangle)$, and that $\mathcal{M} \subseteq \mathcal{D}_{d, \Delta}$. Then $\mathcal{M}$ is said to be a determining set for $\mathcal{S}$ if setting the coefficients of $s \in \mathcal{S}$ associated with the domain points in $\mathcal{M}$ to zero implies that all coefficients of $s$ corresponding to domain points in $\mathcal{D}_{d, \triangle}$ are zero. $\mathcal{M}$ is called a minimal determining set (MDS) for $\mathcal{S}$ if no proper subset $\mathcal{M}^{\prime} \subset \mathcal{M}$ is a determining set. It is well known that $\mathcal{M}$ is an MDS if and only if, given any real numbers $\left\{c_{\xi}\right\}_{\xi \in \mathcal{M}}$, there exists a unique spline $s \in \mathcal{S}$ whose B-coefficients in $\mathcal{M}$ are $\left\{c_{\xi}\right\}_{\xi \in \mathcal{M}}$.

In this paper we are interested in subspaces of $\mathcal{S}_{d}^{0}(\triangle)$ which are defined by requiring that the B-coefficients $\left\{c_{\xi}\right\}_{\xi \in \mathcal{D}_{d, \Delta}}$ satisfy smoothness conditions across edges or at vertices. If $\mathcal{M}$ is a MDS for such a spline space $\mathcal{S}$, then setting the B-coefficients $c_{\xi}$ for all $\xi \in \mathcal{M}$, we can compute all remaining B-coefficients using the smoothness conditions. Indeed, suppose $s$ is a spline in $\mathcal{S}_{d}^{0}(\triangle)$, and let $T:=$ $\left\langle v_{1}, v_{2}, v_{3}\right\rangle$ and $\widetilde{T}:=\left\langle v_{4}, v_{3}, v_{2}\right\rangle$ be a pair of adjoining triangles in $\triangle$ sharing the edge $e=\left\langle v_{2}, v_{3}\right\rangle$. Let $c_{i j k}$ and $\tilde{c}_{i j k}$ be the B-coefficients of $\left.s\right|_{T}$ and $\left.s\right|_{\widetilde{T}}$, respectively. Then it is well known (cf. [12,22]) that $s$ is $C^{r}$ continuous across the edge $e$ if and only if

$$
\tilde{c}_{n, m-n, d-m}=\sum_{i+j+k=n} c_{i, j+d-m, k+m-n} B_{i j k}^{n}\left(v_{4}\right)
$$

for $m=n, \ldots, d$ and $n=1, \ldots, r$. Here $B_{i j k}^{n}$ are the Bernstein polynomials of degree $n$ on the triangle $T$. We recall the following two lemmas from [25]. 
Lemma 4.1. Suppose $s$ is a spline in $\mathcal{S}_{d}^{r}(\triangle)$, and that for fixed $n \leq m \leq d$ and $1 \leq n \leq r$ the coefficients appearing on the right-hand side of (4.1) are known, and that $C$ is the maximum of their absolute values. Then $\left|c_{n, m-n, d-m}\right| \leq K C$, where $K$ is a constant depending only on $d$ and the smallest angle in the triangulation.

Lemma 4.2. Suppose $T$ and $\widetilde{T}$ are two triangles as above, and suppose

$$
v_{4}=\alpha v_{1}+\beta v_{2}+\gamma v_{3}
$$

with $\gamma \neq 0$. Suppose we know the coefficients of a spline $s \in \mathcal{S}_{d}^{r}(\triangle)$ for all domain points in the disk $D_{m-1}\left(v_{2}\right)$ with $m \geq r+1$. Let $c_{i}:=c_{i, d-m, m-i}^{T}$ be the coefficients of $p:=\left.s\right|_{T}$ on the arc $a_{m, e}^{r}\left(v_{2}\right)$ associated with the edge $e:=\left\langle v_{2}, v_{3}\right\rangle$, and let

$\tilde{c}_{i}:=c_{i, m-i, d-m}^{\widetilde{T}}$ be those of $\tilde{p}:=\left.s\right|_{\widetilde{T}}$ on the same arc. Suppose that the coefficients $c_{i}$ and $\tilde{c}_{i}$ are known for $i \in\{r-q+1, \ldots, r\}$ and that the coefficients $c_{0}, \ldots, c_{r-2 q}$ are also known for some $q$ with $r+1 \geq 2 q$. Then the coefficients $c_{i}$ and $\tilde{c}_{i}$ are uniquely determined for all $0 \leq i \leq r$. Moreover, if $C$ is the maximum of the known coefficients, then the computed coefficients are bounded by $K C$, where $K$ is a constant depending only on $d$, the smallest angle in the triangulation, and the size of $\gamma^{-1}$.

Lemma 4.2 cannot be applied in the situation when $\gamma=0$. In this case the edge $e:=\left\langle v_{2}, v_{3}\right\rangle$ is called degenerate. The stability of the computation is also compromised whenever $e$ is near-degenerate in the sense that $\gamma$ is near zero. We discuss near-degenerate edges in more detail in Sect. 7.

\section{$\S 5$. Dual bases}

Our construction of stable local bases will be based on the following

Lemma 5.1. [6] Suppose $\mathcal{M}$ is a $M D S$ for a space of splines $\mathcal{S}$ which is a subset of $\mathcal{S}_{d}^{0}(\triangle)$ defined by smoothness conditions. For each $\xi \in \mathcal{M}$, let $B_{\xi}$ be the unique spline in $\mathcal{S}$ satisfying

$$
\lambda_{\eta} B_{\xi}=\delta_{\xi, \eta}, \quad \text { all } \eta \in \mathcal{M}
$$

where $\lambda_{\eta}$ is the linear functional which picks off the B-coefficient $c_{\eta}$. Then the set $\left\{B_{\xi}\right\}_{\xi \in \mathcal{M}}$ is a basis for $\mathcal{S}$. We call it the dual basis corresponding to $\mathcal{M}$.

Proof: To construct the spline $B_{\xi}$, choose $c_{\xi}=1$, and set all other coefficients corresponding to $\eta \in \mathcal{M}$ to zero. Then since $\mathcal{M}$ is a MDS, all remaining coefficients are uniquely determined. Property (5.1) is obvious.

For a given spline space $\mathcal{S}$, there are generally many different minimal determining sets $\mathcal{M}$. Our aim in this paper is to design algorithms for choosing minimal determining sets which produce stable local bases when applied to a space $\mathcal{S} \subseteq \mathcal{S}_{d}^{0}(\triangle)$. 
Definition 5.2. Suppose $\mathcal{B}:=\left\{B_{\xi}\right\}_{\xi \in \mathcal{M}}$ is a basis for a spline space $\mathcal{S} \subseteq \mathcal{S}_{d}^{0}(\triangle)$. Then we call $\mathcal{B}$ a stable local basis provided that there exist constants $\ell$ and $K$ depending only on $d$ and the smallest angle $\theta_{\triangle}$ in $\triangle$ such that

$$
\begin{gathered}
\operatorname{supp}\left(B_{\xi}\right) \subseteq \operatorname{star}^{\ell}\left(v_{\xi}\right) \text { for some vertex } v_{\xi}, \\
\left\|B_{\xi}\right\|_{\infty} \leq K
\end{gathered}
$$

for all $\xi \in \mathcal{M}$. A minimal determining set $\mathcal{M}$ for a spline space $\mathcal{S}$ is called a stable local MDS provided that the corresponding dual basis $\mathcal{B}:=\left\{B_{\xi}\right\}_{\xi \in \mathcal{M}}$ is a stable local basis for $\mathcal{S}$.

For (5.2), we recall that given a vertex $v$ of $\triangle, \operatorname{star}(v)=\operatorname{star}^{1}(v)$ is the union of triangles sharing $v$, and $\operatorname{star}^{\ell}(v), \ell \geq 2$, is defined recursively as the union of the stars of the vertices in $\mathcal{V} \cap \operatorname{star}^{\ell-1}(v)$.

Theorem 5.3. Suppose that $\mathcal{M}$ is a stable local minimal determining set for a spline space $\mathcal{S} \subseteq \mathcal{S}_{d}^{0}(\triangle)$. Then the dual basis $\mathcal{B}$ satisfies (1.2).

Proof: Let $s \in \mathcal{S}$. Then for any $\xi \in \mathcal{M}$, the corresponding coefficient $c_{\xi}$ is the B-coefficient of the polynomial $s_{T}:=\left.s\right|_{T}$, where $T$ is a triangle containing $\xi$. But then by the stability of the Bernstein-Bézier basis for polynomials (cf. Lemma 4.1 of [25]), we have $\left|c_{\xi}\right| \leq C\left\|s_{T}\right\|_{\infty, T} \leq C\|s\|_{\infty}$, where $C$ is a constant which depends only on $d$. This establishes the left-hand side of (1.2) with the constant $K_{1}:=1 / C$ which depends only on $d$. For the right-hand side, we note that by Lemma 3.1 of [25], for any triangle $T$, the number of basis splines $B_{\xi}$ whose supports contain $T$ is bounded by a constant depending only on $d$ and $\theta_{\triangle}$ (cf. the proof of Theorem 9.2 in [25]). Combining this with the boundedness of the basis functions completes the proof.

With an appropriate renorming, it can be shown that the dual splines also provide a basis which is $L_{p}$-stable for $1 \leq p<\infty$, see Remark 13.7.

\section{$\S 6$. Four-cells}

In this section we construct minimal determining sets for spline spaces defined on triangulations which consist of exactly four triangles surrounding one interior vertex $v$. We call such a triangulation $\triangle_{v}$ a four-cell. We are particularly interested in the case where the vertex is singular (formed by the intersection of two straight lines) or near-singular.

Suppose $v_{1}, \ldots, v_{4}$ are the boundary vertices of $\triangle_{v}$ in counterclockwise order. Let $T_{i}:=\left\langle v, v_{i}, v_{i+1}\right\rangle$, for $i=1, \ldots, 4$, where $v_{5}=v_{1}$. For $\mu+1 \leq \ell \leq 2 r$, we introduce some simplified notation for certain domain points on the ring $R_{\ell}(v)$. Let

$$
\begin{aligned}
& a_{\ell, j}^{i}:=\xi_{d-\ell, \ell-r+j-1, r-j+1}^{T_{i}}, \quad 1 \leq j \leq n_{\ell}, \\
& g_{\ell, j}^{i}:=\xi_{d-\ell, \ell-r+n_{\ell}+j-1, r-n_{\ell}-j+1}^{T_{i}}, \quad 1 \leq j \leq n_{\ell}, \\
& d_{\ell, j}^{i}:=\xi_{d-\ell, \ell-r+2 n_{\ell}+j-1, r-2 n_{\ell}-j+1}^{T_{i}}, \quad 1 \leq j \leq r-2 n_{\ell}+1,
\end{aligned}
$$




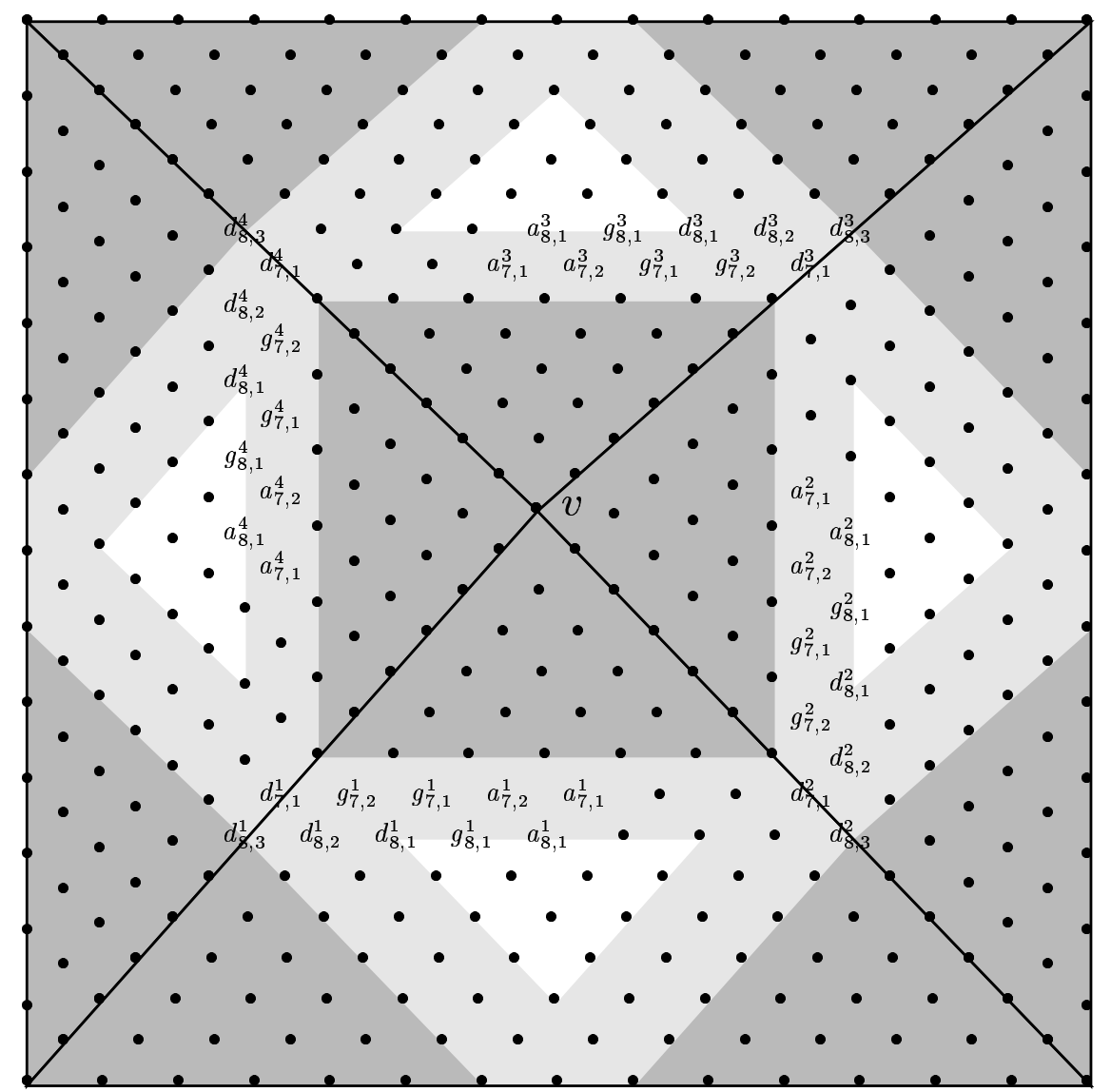

$v_{1}$

Fig. 1. The points in (6.1) for $r=4, \mu=6, d=14$.

where

$$
n_{\ell}:=2 r+1-\ell
$$

Note that $n_{\ell} \geq 1$ and $r-2 n_{\ell}+1 \geq 1$. We illustrate this notation for $r=4, \mu=6$, and $d=14$ in Fig. 1 .

Let $1 \leq k \leq 2 r$. We say that $\mathcal{M} \subseteq D_{k}(v)$ is a MDS for $\mathcal{S}_{d}^{r, \mu}\left(\triangle_{v}\right)$ on $D_{k}(v)$ if setting the coefficients $\left\{c_{\xi}: \xi \in \mathcal{M}\right\}$ to arbitrary real numbers, the coefficients $\left\{c_{\xi}\right.$ : $\left.\xi \in D_{k}(v) \backslash \mathcal{M}\right\}$ can be uniquely computed by using those smoothness conditions defining $\mathcal{S}_{d}^{r, \mu}\left(\triangle_{v}\right)$ that do not involve $c_{\xi}$ for $\xi \notin D_{k}(v)$. Such an MDS will be called stable if

$$
\max _{\xi \in D_{k}(v) \backslash \mathcal{M}}\left|c_{\xi}\right| \leq K \max _{\xi \in \mathcal{M}}\left|c_{\xi}\right|
$$

where $K$ is a constant depending only on $d$ and the smallest angle $\theta_{\triangle}$ in $\triangle_{v}$. By Lemma 4.1 it is easy to see that $D_{k}^{T_{1}}(v)$ is a stable MDS for $\mathcal{S}_{d}^{r, \mu}\left(\triangle_{v}\right)$ on $D_{k}(v)$ if 
$k \leq \mu$.

It is not difficult to describe a stable MDS for $\mathcal{S}_{d}^{r, \mu}\left(\triangle_{v}\right)$ on $D_{k}(v)$ for $\mu+1 \leq$ $k \leq 2 r$ if $v$ is singular.

Theorem 6.1. Suppose $\triangle_{v}$ is a four-cell associated with a singular vertex $v$. For each $\ell=\mu+1, \ldots, 2 r$, let

$$
\begin{aligned}
\mathcal{M}_{v, \ell} & :=\left\{a_{\ell, 1}^{1}, \ldots, a_{\ell, n_{\ell}}^{1}\right\} \cup \bigcup_{i=1}^{4}\left\{g_{\ell, 1}^{i}, \ldots, g_{\ell, n_{\ell}}^{i}\right\}, \\
O_{v, \ell} & :=\bigcup_{i=1}^{4}\left\{d_{\ell, 1}^{i}, \ldots, d_{\ell, r-2 n_{\ell}+1}^{i}\right\} .
\end{aligned}
$$

Then for each $k=\mu, \ldots, 2 r$,

$$
\Gamma_{k}:=D_{\mu}^{T_{1}}(v) \cup \bigcup_{\ell=\mu+1}^{k}\left[\mathcal{M}_{v, \ell} \cup O_{v, \ell}\right]
$$

is a stable $M D S$ for the space $\mathcal{S}_{d}^{r, \mu}\left(\triangle_{v}\right)$ on $D_{k}(v)$.

Proof: We proceed by induction on $k$. The result is clear for $k=\mu$. Suppose we set the coefficients $c_{\xi}$ of $s \in \mathcal{S}_{d}^{r, \mu}\left(\triangle_{v}\right)$ for $\xi \in \Gamma_{k}$. Then by the inductive hypothesis, all coefficients $c_{\xi}$ with $\xi \in D_{k-1}(v)$ are uniquely determined by those with $\xi \in \Gamma_{k-1} \subseteq \Gamma_{k}$. We then compute the coefficients associated with domain points on the ring $R_{k}(v)$ using the standard smoothness conditions as in Lemma 4.1. Namely, for each $i=1,2,3,4$, we first use the coefficients associated with the domain points $g_{k, 1}^{i+1}, \ldots, g_{k, n_{k}}^{i+1}, d_{k, 1}^{i+1}, \ldots, d_{k, r-2 n_{k}+1}^{i+1}$ and those in $D_{k-1}(v)$ to compute the coefficients corresponding to $\left\{\xi_{d-k, 0, k}^{T_{i}}, \ldots, \xi_{d-k, r-n_{k}, k-r+n_{k}}^{T_{i}}\right\}$. After this only the coefficients associated with domain points $a_{k, 1}^{i}, \ldots, a_{k, n_{k}}^{i}, i=2,3,4$, remain undetermined in $R_{k}(v)$. We therefore use the coefficients corresponding to $R_{k}^{T_{1}}(v)$ to compute the coefficients $c_{\xi}$ with $\xi \in\left\{a_{k, 1}^{2}, \ldots, a_{k, n_{k}}^{2}\right\}$, and, proceeding counterclockwise around $v$, successively compute the coefficients with $\xi \in\left\{a_{k, 1}^{3}, \ldots, a_{k, n_{k}}^{3}\right\}$ and $\xi \in\left\{a_{k, 1}^{4}, \ldots, a_{k, n_{k}}^{4}\right\}$. Note that here we have not used a portion of the smoothness conditions across the edge $e_{1}:=\left\langle v, v_{1}\right\rangle$ which involve the coefficients $c_{\xi}$ for $\xi \in\left\{a_{k, 1}^{1}, \ldots, a_{k, n_{k}}^{1}\right\}$. Nevertheless, these conditions must be satisfied since the number of free parameters $c_{\xi}, \xi \in \Gamma_{k} \backslash \Gamma_{k-1}$, used in the above computation on ring $R_{k}(v)$, is equal to

$$
\operatorname{dim} \mathcal{S}_{k}^{r, \mu}\left(\triangle_{v}\right)-\operatorname{dim} \mathcal{S}_{k-1}^{r, \mu}\left(\triangle_{v}\right)=4(k-r)+n_{k}
$$

(cf. Theorem 2.2 of [30]). Thus, we are able to compute all coefficients $c_{\xi}, \xi \in$ $D_{k}(v) \backslash \Gamma_{k}$, by applying Lemma 4.1 several times. By that lemma, the maximum of the computed coefficients is bounded by a constant $K$ times the maximum of the set coefficients, where $K$ depends only on $d$ and the smallest angle in $\triangle_{v}$. 
For later use in building stable local minimal determining sets for general spline spaces, it is critical that the stable MDS in Theorem 6.1 contains the sets $O_{v, \ell}$. We now extend this result to arbitrary four-cells, although in fact we will use it only for cells which are near-singular. In Sect. 10 we construct stable minimal determining sets for supersplines on general cells. The construction there is simpler, but does not guarantee that the resulting MDS contains the needed sets $O_{v, \ell}$.

Theorem 6.2. Suppose $\triangle_{v}$ is a four-cell associated with a nonsingular vertex $v$. For each $\mu+1 \leq \ell \leq 2 r$, let $O_{v, \ell}$ be the sets in (6.3), and let $n_{\ell}$ be the integer defined in (6.2). Then there exists a set of $4 n_{\ell}$ domain points

$$
\mathcal{M}_{v, \ell} \subseteq \mathcal{A}_{v, \ell}:=\bigcup_{i=1}^{4}\left[\left\{a_{\ell, j}^{i}\right\}_{j=1}^{n_{\ell}} \cup\left\{g_{\ell, j}^{i}\right\}_{j=1}^{n_{\ell}}\right]
$$

such that for each $k=\mu, \ldots, 2 r$,

$$
\Gamma_{k}:=D_{\mu}^{T_{1}}(v) \cup \bigcup_{\ell=\mu+1}^{k}\left[\mathcal{M}_{v, \ell} \cup O_{v, \ell}\right]
$$

is a stable MDS for the space $\mathcal{S}_{d}^{r, \mu}\left(\triangle_{v}\right)$ on $D_{k}(v)$.

Proof: We proceed by induction on $k$. The statement of the theorem holds for $k=\mu$ since $\Gamma_{\mu}=D_{\mu}^{T_{1}}(v)$ is trivially a stable MDS for $\mathcal{S}_{d}^{r, \mu}\left(\triangle_{v}\right)$ on $D_{\mu}(v)$.

Fix $\mu+1 \leq k \leq 2 r$, and suppose that $\Gamma_{k-1}$ is a stable MDS for $\mathcal{S}_{d}^{r, \mu}\left(\triangle_{v}\right)$ on $D_{k-1}(v)$. To construct $\Gamma_{k}$ which is a stable MDS for $\mathcal{S}_{d}^{r, \mu}\left(\triangle_{v}\right)$ on $D_{k}(v)$, we need to supplement $\Gamma_{k-1}$ with an appropriate subset of the domain points on the ring $R_{k}(v)$. Using the fact that $v$ is not a singular vertex, it is easy to see that the number of edges attached to $v$ with different slopes is at least three. Then Theorem 2.2 of [30] implies

$$
m:=\operatorname{dim} \mathcal{S}_{k}^{r, \mu}\left(\triangle_{v}\right)-\operatorname{dim} \mathcal{S}_{k-1}^{r, \mu}\left(\triangle_{v}\right)=4(k-r)
$$

Thus, to get a minimal determining set $\Gamma_{k}$ for $\mathcal{S}_{d}^{r, \mu}\left(\triangle_{v}\right)$ on $D_{k}(v)$, we need to add to $\Gamma_{k-1}$ exactly $m$ points on the ring $R_{k}(v)$.

To simplify the discussion of how to choose these $m$ points, we first reduce the problem to one of considering splines whose coefficients are zero for all points in the disk $D_{k-1}(v)$. Given $s \in \mathcal{S}_{d}^{r, \mu}\left(\triangle_{v}\right)$, let $\mathcal{T}_{k-1} s$ be the spline in $\mathcal{S}_{d}^{r}\left(\triangle_{v}\right)$ constructed in Lemma 6.4 below such that for each triangle attached to $v, g_{T}:=\left.\mathcal{T}_{k-1} s\right|_{T}$ interpolates the derivatives up to order $k-1$ of $\left.s\right|_{T}$ at $v$. Note that since $s \in C^{\mu}(v)$, $\mathcal{T}_{k-1} s$ is also in $C^{\mu}(v)$. Then the spline $\hat{s}:=s-\mathcal{T}_{k-1} s \in \mathcal{S}_{d}^{r, \mu}\left(\triangle_{v}\right)$ has all zero coefficients in $D_{k-1}(v)$. Computing its coefficients on the ring $R_{k}(v)$ will stably and uniquely determine the coefficients of $s$ on $R_{k}(v)$, since by Lemma 6.4 the size of the coefficients of $\mathcal{T}_{k-1} s$ on this ring is bounded by the size of the coefficients of $s$ in $D_{k-1}(v)$. 
We now focus on the set $\mathcal{A}_{v, k} \cup O_{v, k}$ of domain points $a_{k, j}^{i}, g_{k, j}^{i}$, and $d_{k, j}^{i}$ lying on ring $R_{k}(v)$. Clearly, if we are given values for the coefficients $c_{\xi}$ of $\hat{s}$ for $\xi \in \mathcal{A}_{v, k} \cup O_{v, k}$, then the remaining coefficients of $\hat{s}$ corresponding to domain points on $R_{k}(v) \backslash\left(\mathcal{A}_{v, k} \cup O_{v, k}\right)$ can be computed directly and stably from smoothness conditions using Lemma 4.1.

Suppose $v_{1}, \ldots, v_{4}$ are the boundary vertices of $\triangle_{v}$ in counterclockwise order, and let $e_{i}:=\left\langle v, v_{i}\right\rangle$ and $T_{i}:=\left\langle v, v_{i}, v_{i+1}\right\rangle$ for $i=1, \ldots, 4$, where for convenience we identify $v_{i+4}$ with $v_{i}$ for all $i$. In addition, suppose the barycentric coordinates of $v_{i-1}$ with respect to the triangle $T_{i}$ are given by

$$
v_{i-1}=r_{i} v_{i+1}+s_{i} v+t_{i} v_{i}
$$

for $i=1,2,3,4$. Note that $t_{i}=0$ if and only if the edge $e_{i}$ is degenerate at $v$. Since $v$ is assumed not to be a singular vertex, at least one $t_{i}$ is nonzero. points

Let $z=\left(z_{1}, \ldots, z_{4 r+4}\right)$ be the B-coefficients of $\hat{s}$ corresponding to the domain

$$
\bigcup_{i=1}^{4}\left\{a_{k, 1}^{i}, \ldots, a_{k, n}^{i}, g_{k, 1}^{i}, \ldots, g_{k, n}^{i}, d_{k, 1}^{i}, \ldots, d_{k, r-2 n+1}^{i}\right\} .
$$

Here we are writing $n:=n_{k}=2 r+1-k$ for ease of notation. Since the coefficients of $\hat{s}$ corresponding to domain points in $D_{k-1}(v)$ are zero, the smoothness conditions of order $r-n+1, \ldots, r$ across the interior edges of $\triangle_{v}$ which connect the components of $z$ to each other can be written in the form

$$
H z=0,
$$

where

$$
\begin{aligned}
& H:=\left(\begin{array}{ccccccccccccc}
H_{1}^{a} & H_{1}^{g} & H_{1}^{d} & & & & & & & -I & & \\
-I & & & H_{2}^{a} & H_{2}^{g} & H_{2}^{d} & & & & & & \\
& & & -I & & & H_{3}^{a} & H_{3}^{g} & H_{3}^{d} & & & \\
& & & & & & -I & & & H_{4}^{a} & H_{4}^{g} & H_{4}^{d}
\end{array}\right),
\end{aligned}
$$

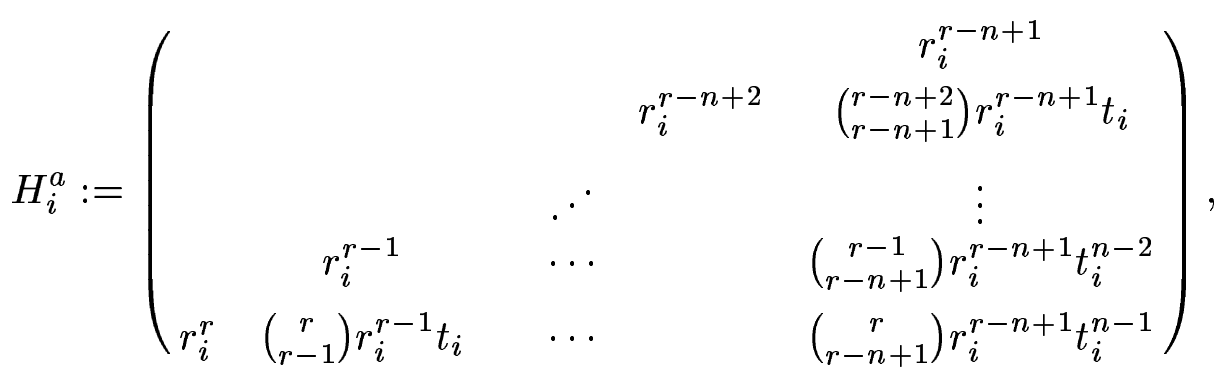

$$
\begin{aligned}
& H_{i}^{g}:=\left(\begin{array}{ccc}
\left(\begin{array}{c}
r-n+1 \\
r-n
\end{array}\right) r_{i}^{r-n} t_{i} & \cdots & \left(\begin{array}{c}
r-n+1 \\
r-2 n+1
\end{array}\right) r_{i}^{r-2 n+1} t_{i}^{n} \\
\vdots & & \vdots \\
\left(\begin{array}{c}
r \\
r-n
\end{array}\right) r_{i}^{r-n} t_{i}^{n} & \cdots & \left(\begin{array}{c}
r \\
r-2 n+1
\end{array}\right) r_{i}^{r-2 n+1} t_{i}^{2 n-1}
\end{array}\right),
\end{aligned}
$$




$$
H_{i}^{d}:=\left(\begin{array}{cccc}
\left(\begin{array}{c}
r-n+1 \\
r-2 n
\end{array}\right) r_{i}^{r-2 n} t_{i}^{n+1} & \ldots & \left(\begin{array}{c}
r-n+1 \\
1
\end{array}\right) r_{i} t_{i}^{r-n} & t_{i}^{r-n+1} \\
\vdots & & \vdots & \vdots \\
\left(\begin{array}{c}
r \\
r-2 n
\end{array}\right) r_{i}^{r-2 n} t_{i}^{2 n} & \ldots & \left(\begin{array}{c}
r \\
1
\end{array}\right) r_{i} t_{i}^{r-1} & t_{i}^{r}
\end{array}\right),
$$

and $I$ is the $n \times n$ identity matrix. We call a column of $H$ a $d$-column when it passes through one of the matrices $H_{i}^{d}$. We define $a$-columns and $g$-columns similarly.

The matrix $H$ has $4 n$ rows and $4(r+1)$ columns where $n<r+1$. We claim that it has full rank $4 n$. Indeed, the number of independent solutions $4(r+1)-\operatorname{rank}(H)$ of the homogeneous system (6.8) must be equal to $m$, which implies $\operatorname{rank}(H)=4 n$. This means that there is a choice of $4 n$ indices $1 \leq i_{1}<\cdots<i_{4 n} \leq 4 r+4$ such that the determinant of the corresponding square submatrix $H\left(i_{1}, \cdots, i_{4 n}\right)$ is not zero. Moreover, it follows from Lemma 6.3 below that we can choose $i_{1}, \cdots, i_{4 n}$ such that no column of $H\left(i_{1}, \cdots, i_{4 n}\right)$ is a $d$-column.

We are ready to describe the set $\mathcal{M}_{v, k}$. Denote by $\Sigma$ the set of indices of all $a$ - and $g$-columns of $H$, and let $\left\{i_{1}^{*}, \cdots, i_{4 n}^{*}\right\} \subseteq \Sigma$ be such that

$$
\left|\operatorname{det} H\left(i_{1}^{*}, \ldots, i_{4 n}^{*}\right)\right|=\max _{i_{1}, \ldots, i_{4 n} \in \Sigma}\left|\operatorname{det} H\left(i_{1}, \ldots, i_{4 n}\right)\right| .
$$

We take $\mathcal{M}_{v, k}$ to be the set of domain points in $\mathcal{A}_{v, k}$ which correspond to the columns with indices in the set $\Sigma \backslash\left\{i_{1}^{*}, \cdots, i_{4 n}^{*}\right\}$. Then $\mathcal{M}_{v, k} \cup \mathcal{O}_{v, k}$ is the set of domain points on $R_{k}(v)$ which correspond to the columns of $H$ with indices in the set $J^{*}:=\{1, \ldots, 4 r+4\} \backslash\left\{i_{1}^{*}, \cdots, i_{4 n}^{*}\right\}$.

Now assuming that the coefficients $\left\{z_{j}\right\}_{j \in J^{*}}$ of $\hat{s}$ corresponding to points in $\mathcal{M}_{v, k} \cup \mathcal{O}_{v, k}$ have been set, we may compute the remaining coefficients corresponding to points in $\mathcal{A}_{v, k} \cup \mathcal{O}_{v, k}$ from the nonsingular system

$$
H\left(i_{1}^{*}, \ldots, i_{4 n}^{*}\right)\left(\begin{array}{c}
z_{i_{1}^{*}} \\
\vdots \\
z_{i_{4 n}^{*}}
\end{array}\right)=-\sum_{j \in J^{*}} z_{j} H(j)
$$

where $H(j)$ is the $j$-th column of $H$. Using Cramer's rule and taking account of (6.9) and Lemma 6.3, we conclude that

$$
\left|z_{i_{\nu}^{*}}\right| \leq \frac{\sum_{j \in J^{*}}\left|z_{j}\right|\left|\operatorname{det} H\left(i_{1}^{*}, \ldots, i_{\nu-1}^{*}, j, i_{\nu+1}^{*}, \ldots, i_{4 n}^{*}\right)\right|}{\left|\operatorname{det} H\left(i_{1}^{*}, \ldots, i_{4 n}^{*}\right)\right|} \leq K \max _{j \in J^{*}}\left|z_{j}\right|,
$$

for $\nu=1, \ldots, 4 n$, where $K$ is a constant depending only on $d$ and the smallest angle in $\triangle_{v}$. This shows that the computation of $z_{i_{1}^{*}}, \ldots, z_{i_{4 n}^{*}}$ is stable.

We now state and prove two lemmas which were used in the proof of Theorem 6.2. The first result concerns determinants formed from $4 n \times 4 n$ submatrices of $H$. Let

$$
R:=\left(\begin{array}{ccc}
\left(\begin{array}{c}
r-n+1 \\
r-n
\end{array}\right) & \cdots & \left(\begin{array}{c}
r-n+1 \\
r-2 n+1
\end{array}\right) \\
\vdots & \ddots & \vdots \\
\left(\begin{array}{c}
r \\
r-n
\end{array}\right) & \cdots & \left(\begin{array}{c}
r \\
r-2 n+1
\end{array}\right)
\end{array}\right)
$$


A simple computation shows that

$$
\operatorname{det} R=C \operatorname{det}\left(\begin{array}{ccc}
\frac{1}{1 !} & \cdots & \frac{1}{n !} \\
\vdots & \cdots & \vdots \\
\frac{1}{n !} & \cdots & \frac{1}{(2 n-1) !}
\end{array}\right)
$$

where $C$ is a positive constant depending only on $r$ and $n$. It is well-known that this determinant is nonzero for all choices of $n$, and thus the matrix $R$ is nonsingular.

Lemma 6.3. Let $H\left(i_{1}, \ldots, i_{4 n}\right)$ be a $4 n \times 4 n$ submatrix of $H$ containing a nontrivial $d$-column. Then there exists another submatrix $H\left(j_{1}, \ldots, j_{4 n}\right)$ with one less $d$ column such that

$$
\left|\operatorname{det} H\left(i_{1}, \ldots, i_{4 n}\right)\right| \leq C\left|\operatorname{det} H\left(j_{1}, \ldots, j_{4 n}\right)\right|,
$$

where $C>0$ is a constant depending only on $d$ and the smallest angle of $\triangle_{v}$.

Proof: Suppose $H\left(i_{1}, \ldots, i_{4 n}\right)$ includes a nontrivial $d$-column $i_{p}=(r+1)(i-1)+$ $2 n+j$ with $1 \leq i \leq 4$ and $1 \leq j \leq r-2 n+1$. Note that the column is nontrivial if and only if the corresponding $t_{i}$ is nonzero. For any $1 \leq j \leq r-2 n+1$, it is not difficult to see that

$$
H_{i}^{d}(j)=\sum_{\kappa=1}^{n} x_{\kappa}^{[j]}\left(\frac{t_{i}}{r_{i}}\right)^{j+n-\kappa} H_{i}^{g}(\kappa),
$$

where the numbers $x_{\kappa}^{[j]}$ are determined from the nonsingular linear system

$$
R\left(\begin{array}{c}
x_{1}^{[j]} \\
\vdots \\
x_{n}^{[\nu]}
\end{array}\right)=\left(\begin{array}{c}
\left(\begin{array}{c}
r-n+1 \\
r-2 n+1-j
\end{array}\right) \\
\vdots \\
r \\
r-2 n+1-j
\end{array}\right) .
$$

Since the $\kappa$-th column of $H_{i}^{g}$ corresponds to the $(r+1)(i-1)+n+\kappa$-th column of $H$, this implies that

$$
\operatorname{det} H\left(i_{1}, \ldots, i_{4 n}\right)=\sum_{\kappa=1}^{n} x_{\kappa}^{[j]}\left(\frac{t_{i}}{r_{i}}\right)^{j+n-\kappa} \operatorname{det} H_{\kappa},
$$

where

$$
H_{\kappa}:=H\left(i_{1}, \ldots, i_{p-1},(r+1)(i-1)+n+\kappa, i_{p+1}, \ldots, i_{4 n}\right) .
$$

Since $\left|r_{i}\right|$ is the quotient of the areas of two neighboring triangles $T_{i-1}$ and $T_{i}$, we have by Lemma 3.2 of [25],

$$
0<K_{1} \leq\left|r_{i}\right| \leq K_{2},
$$

where $K_{1}, K_{2}$ depend only on $\theta_{\triangle}$. Therefore,

$$
\left|\operatorname{det} H\left(i_{1}, \ldots, i_{4 n}\right)\right| \leq K_{3}\left|t_{i}\right|^{j} \max _{\kappa}\left|\operatorname{det} H_{\kappa}\right|,
$$

where $K_{3}$ depends only on $d$ and $\theta_{\triangle}$. The result follows since $\left|t_{i}\right| \leq K_{4}$ where $K_{4}$ is a constant depending only on $\theta_{\triangle}$. (In fact, $\left|t_{i}\right|$ is quite small if $v$ is near-singular.)

The following lemma was used in the proof of Theorem 6.2 above, and will also be useful in Sect. 12 below. 
Lemma 6.4. Let $\triangle_{v}$ be a cell, and let $0 \leq r<k \leq d$ be integers. Given a spline $s \in \mathcal{S}_{d}^{r}\left(\triangle_{v}\right)$, let $\mathcal{T}_{k-1} s$ be such that for each triangle $T$ attached to $v$,

$\left.\mathcal{T}_{k-1} s\right|_{T}:=$ the unique polynomial of degree $k-1$ which matches

the derivatives of $\left.s\right|_{T}$ at $v$ up to order $k-1$.

Then $\mathcal{T}_{k-1} s \in \mathcal{S}_{k-1}^{r}\left(\triangle_{v}\right) \subseteq \mathcal{S}_{d}^{r}\left(\triangle_{v}\right)$. Moreover, if

$$
\left.s\right|_{T}=\sum c_{\xi}^{T} B_{\xi}^{T},\left.\quad \mathcal{T}_{k-1} s\right|_{T}=\sum \hat{c}_{\xi}^{T} B_{\xi}^{T}
$$

where $B_{\xi}^{T}$ are the Bernstein polynomials of degree $d$ associated with a triangle $T$, then $\hat{c}_{\xi}^{T}=c_{\xi}^{T}$ for all $\xi \in D_{k-1}^{T}(v)$, and

$$
\max _{\xi \in R_{k}^{T}(v)}\left|\hat{c}_{\xi}^{T}\right| \leq K \max _{\xi \in D_{k-1}^{T}(v)}\left|c_{\xi}^{T}\right|
$$

where $K$ is a constant depending only on $d$.

Proof: Comparing cross derivatives of neighboring pieces of $\mathcal{T}_{k-1} s$, it is easy to see that it satisfies $C^{r}$ smoothness conditions across the interior edges of $\triangle_{v}$, and thus is a spline in $\mathcal{S}_{k-1}^{r}\left(\triangle_{v}\right) \subseteq \mathcal{S}_{d}^{r}(\triangle)$. Now fix a triangle $T:=\left\langle v, v_{i}, v_{i+1}\right\rangle$ in $\triangle_{v}$. Then by the well-known connection between derivatives and coefficients of a polynomial written in Bernstein-Bézier form, it follows that $\hat{c}_{\xi}^{T}=c_{\xi}^{T}$ for all $\xi \in D_{k-1}^{T}(v)$. Finally, to establish (6.15), we observe that since $\mathcal{T}_{k-1} s$ is a polynomial of degree $k-1$, its $k$-th derivatives are identically zero, and thus for all $\nu=0, \ldots, k$,

$$
\begin{aligned}
0 & =\left.D_{v_{i}-v}^{\nu} D_{v_{i+1}-v}^{k-\nu} \mathcal{T}_{k-1} s\right|_{T}(v) \\
& =\frac{d !}{(d-k) !} \sum_{j_{1}=0}^{\nu} \sum_{j_{2}=0}^{k-\nu}\left(\begin{array}{c}
\nu \\
j_{1}
\end{array}\right)\left(\begin{array}{c}
k-\nu \\
j_{2}
\end{array}\right)(-1)^{k-j_{1}-j_{2}} \hat{c}_{d-j_{1}-j_{2}, j_{1}, j_{2}}^{T} .
\end{aligned}
$$

It follows that

$$
\hat{c}_{d-k, \nu, k-\nu}^{T}=-\frac{d !}{(d-k) !} \sum_{\substack{0 \leq j_{1} \leq \nu, 0 \leq j_{2} \leq k-\nu \\
j_{1}+j_{2} \leq k-1}}\left(\begin{array}{c}
\nu \\
j_{1}
\end{array}\right)\left(\begin{array}{c}
k-\nu \\
j_{2}
\end{array}\right)(-1)^{k-j_{1}-j_{2}} \hat{c}_{d-j_{1}-j_{2}, j_{1}, j_{2}}^{T},
$$

which immediately implies (6.15).

Example 6.5. Let $r=5, \mu=8, d=17$ in Theorem 6.2.

Discussion: Fig. 2 shows the domain points in this case, where the $D_{2 r}$ disks are shaded light gray and the $D_{\mu}$ disks are shaded dark gray. We concentrate on the ring $R_{9}(v)$, where $n_{9}=2$. The points in $O_{v, 9}$ are marked with the symbol $\odot$ (except for the point $d_{9,2}^{3}$ which we have marked with a $\oplus$ for a later discussion). In this case the set $\mathcal{M}_{v, 9}$ must contain eight of the sixteen points in the set $\mathcal{A}_{v, 9}$ described in (6.5). These eight points are chosen by the method of maximization of the determinant in (6.9), and therefore depend on the exact geometry of the cell. In Fig. 2 we show a possible constellation where we mark the eight points in $\mathcal{M}_{v, 9} \cap \mathcal{A}_{v, 9}$ with the symbol $\square$. This leaves eight points which are computed by the linear system (6.8). They are marked with boxed numbers 1 through 8 . 


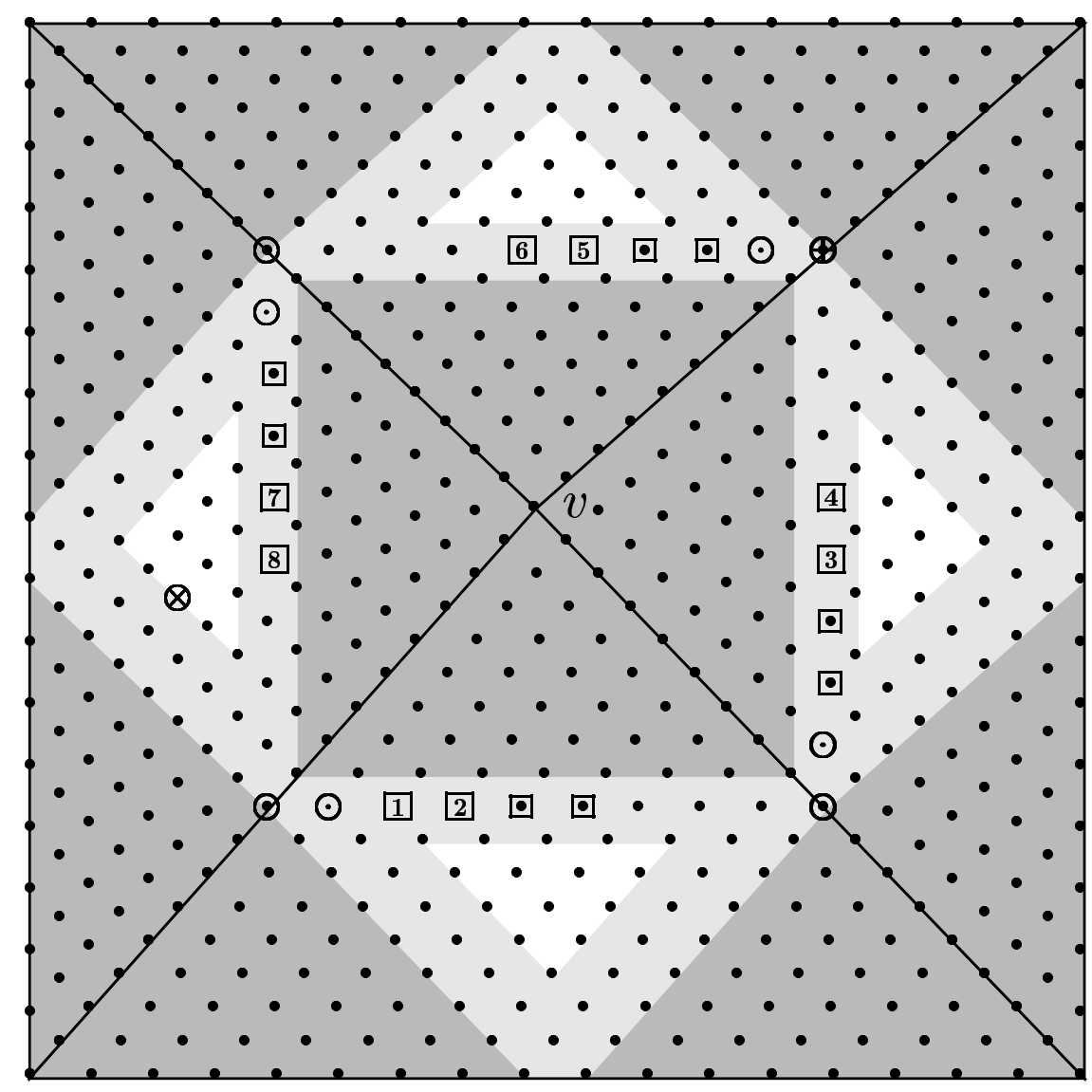

$v_{1}$

Fig. 2. The points in $\mathcal{M} \cap R_{9}(v)$ for $r=5, \mu=8, d=17$.

\section{$\S 7$. Near-degenerate edges}

We recall that an edge $e=\left\langle v_{2}, v_{3}\right\rangle$ shared by two triangles $T:=\left\langle v_{1}, v_{2}, v_{3}\right\rangle$ and $\widetilde{T}:=\left\langle v_{4}, v_{3}, v_{2}\right\rangle$ is called degenerate at $v_{2}$ provided that the points $v_{1}, v_{2}, v_{4}$ lie on a straight line. An edge which is nearly degenerate is called near-degenerate, cf. [25]. We shall introduce the following quantitative form, where the choice of the constant $2 \theta^{2} / \pi$ is motivated by Lemma 8.2 below.

Definition 7.1. Suppose $T:=\left\langle v_{1}, v_{2}, v_{3}\right\rangle$ and $\widetilde{T}:=\left\langle v_{4}, v_{3}, v_{2}\right\rangle$ are two triangles which share the edge $e=\left\langle v_{2}, v_{3}\right\rangle$. We say that $e$ is $\theta$-near-degenerate at $v_{2}$ provided that the smaller of the two angles between the edges $\left\langle v_{2}, v_{1}\right\rangle$ and $\left\langle v_{2}, v_{4}\right\rangle$ is greater than $\pi-2 \theta^{2} / \pi$.

Let $(\alpha, \beta, \gamma)$ be the barycentric coordinates of $v_{4}$ in terms of the triangle $T$, i.e., $v_{4}=\alpha v_{1}+\beta v_{2}+\gamma v_{3}$. The following lemma gives a lower bound on the size of $\gamma$ 
in the case where $e$ is not $\theta$-near-degenerate. This is important since it shows that for such edges $e$, the process of computing coefficients by smoothness conditions across $e$ as in Lemma 4.2 is stable.

Lemma 7.2. Suppose the smallest angle in the triangles $T$ and $\widetilde{T}$ is at least $\theta$, and that $e_{2}:=\left\langle v_{2}, v_{3}\right\rangle$ is not $\theta$-near-degenerate at $v_{2}$. Then $|\gamma| \geq \sin (\theta) \sin \left(2 \theta^{2} / \pi\right)$.

Proof: Let $e_{1}:=\left\langle v_{2}, v_{1}\right\rangle$ and $e_{3}:=\left\langle v_{2}, v_{4}\right\rangle$. Then

$$
|\gamma|=\frac{\left|e_{1}\right|\left|e_{3}\right| \sin (a)}{\left|e_{1}\right|\left|e_{2}\right| \sin \left(\theta_{1}\right)}
$$

where $a$ is the smaller of the two angles between $e_{1}$ and $e_{3}$, and $\theta_{1}$ is the angle between the edges $e_{1}$ and $e_{2}$. It was shown in the proof of Lemma 3.2 of [25] that under the above hypotheses, $\left|e_{3}\right| /\left|e_{2}\right| \geq \sin (\theta)$. But then the result follows from the fact that $|\sin (a)| \geq \sin \left(2 \theta^{2} / \pi\right)$ while $\left|\sin \left(\theta_{1}\right)\right| \leq 1$.

\section{$\S 8$. Near-singular vertices}

A singular vertex is an interior vertex of a triangulation which is formed by the intersection of exactly two lines. It is well known that singular and near-singular vertices complicate the construction of stable bases for spline spaces.

Definition 8.1. Suppose $v$ is a vertex where four edges meet. If all four edges are $\theta$-near-degenerate at $v$, then we call $v$ a $\theta$-near-singular vertex.

The following lemma will be used later.

Lemma 8.2. Fix $\theta \leq \pi / 4$, and let $\triangle$ be a triangulation with smallest angle at least $\theta$. Then for any interior vertex $v$ which is not $\theta$-near-singular, there is at least one edge attached to $v$ which is not $\theta$-near-degenerate at either end.

Proof: Let $v_{1}, \ldots, v_{n}$ be the vertices attached to $v$ in counter-clockwise order. We set $T_{i}:=\left\langle v, v_{i}, v_{i+1}\right\rangle$ and denote by $\theta_{i}, \phi_{i}, \omega_{i}$ the angles of $T_{i}$ at $v, v_{i}, v_{i+1}$, respectively. We distinguish three cases.

Case 1: $n=3$. Consider the edge $e_{1}:=\left\langle v, v_{1}\right\rangle$, and let $\alpha:=\phi_{1}+\omega_{3}$ and $\beta:=\theta_{1}+\theta_{3}$. Then it is clear that $\beta \geq \pi+2 \theta$. Now $\alpha+\beta+\omega_{1}+\phi_{3}=2 \pi$. This implies $\alpha \leq \pi-4 \theta$ since $\omega_{1}, \phi_{3} \geq \theta$.

Case 2: $n=4$. Since $v$ is not $\theta$-near-singular, there is at least one edge attached to $v$ which is not $\theta$-near-degenerate at $v$. Without loss of generality we can assume it is the edge $e_{1}:=\left\langle v, v_{1}\right\rangle$, and that the angle $\beta:=\theta_{1}+\theta_{4}$ is at least $\pi+2 \theta^{2} / \pi$. Then arguing as in Case 1 , we see that $\alpha:=\phi_{1}+\omega_{4} \leq \pi-2 \theta-2 \theta^{2} / \pi \leq \pi-2 \theta$.

Case 3: $n \geq 5$. Consider the edge $e_{1}:=\left\langle v, v_{1}\right\rangle$. Let $\alpha_{i}:=\phi_{i}+\omega_{i-1}$ and $\beta_{i}:=\theta_{i}+\theta_{i-1}$, for $i=1, \ldots, n$ where we identify $\theta_{n+i}=\theta_{i}, \omega_{n+i}=\omega_{i}$. We claim 
that at least three of the $\alpha_{i}$ satisfy $\alpha_{i} \leq \pi-4 \theta /(n-2)$. Indeed, if this were not the case, then

$$
(n-2) \pi=\sum_{i=1}^{n} \alpha_{i}>(n-2)\left(\pi-\frac{4 \theta}{n-2}\right)+4 \theta=(n-2) \pi .
$$

On the other hand, we claim that at most two of the $\beta_{i}$ satisfy $\beta_{i} \geq \pi-\theta / 2$. Suppose to the contrary that there are three, say $\beta_{k}, \beta_{l}, \beta_{m}$. Then at least two of these do not overlap, say $\beta_{k}, \beta_{l}$. But then there are $n-4$ of the angles $\theta_{i}$ which are not covered by $\beta_{k}$ or $\beta_{l}$, which would lead to the contradiction

$$
2 \pi=\beta_{k}+\beta_{l}+(n-4) \theta>2 \pi-\theta+(n-4) \theta \geq 2 \pi .
$$

Now $n \theta \leq 2 \pi$ implies $4 /(n-2) \geq 4 / n \geq 2 \theta / \pi$. We conclude that for one of the edges, $\alpha_{i} \leq \pi-4 \theta /(n-2) \leq \pi-2 \theta^{2} / \pi$ and $\beta_{i} \leq \pi-\theta / 2 \leq \pi-2 \theta^{2} / \pi$. It follows that this edge is not $\theta$-near-degenerate at either end.

\section{$\S$ 9. Stable local bases for $\mathcal{S}_{d}^{r, \mu}(\triangle)$}

Our ultimate aim is to give stable local bases for the general superspline spaces (1.1) defined on arbitrary triangulations $\triangle$ of a polygonal set $\Omega$. However, in order to illustrate the construction in a somewhat simpler setting, in this section we consider the superspline space

$$
\mathcal{S}_{d}^{r, \mu}(\triangle):=\left\{s \in \mathcal{S}_{d}^{r}(\triangle): s \in C^{\mu}(v) \text { for all } v \in \mathcal{V}\right\}
$$

for $d \geq 3 r+2$, where $\mu$ is defined in (1.3). This is the special case of (1.1) with $\rho_{v}=\mu$ for all $v \in \mathcal{V}$. The analogous construction for general superspline spaces requires further analysis of cells (see Sect. 10), and is given in Section 11.

To describe a minimal determining set for $\mathcal{S}_{d}^{r}(\triangle)$ we need some additional notation. Let $\mathcal{V}_{S}$ and $\mathcal{V}_{N S}$ be the sets of vertices of $\triangle$ which are singular and $\theta_{\triangle^{-}}$ near-singular, respectively, where $\theta_{\triangle}$ is the smallest angle in $\triangle$. Given a triangle $T$, we divide the set $\mathcal{D}_{d, T}$ into various subsets. Let

$$
C^{T}:=\left\{\xi_{i j k}^{T}: i>r, j>r, k>r\right\}
$$

and

$$
\begin{aligned}
D_{\mu}^{T}(u) & :=\left\{\xi_{i j k}^{T}: i \geq d-\mu\right\} \\
A^{T}(u) & :=\bigcup_{i=1}^{\left\lfloor\frac{r}{2}\right\rfloor} \bigcup_{j=0}^{i-1}\left\{\xi_{d-2 r+i-1, r-j, r-i+j+1}^{T}\right\}
\end{aligned}
$$




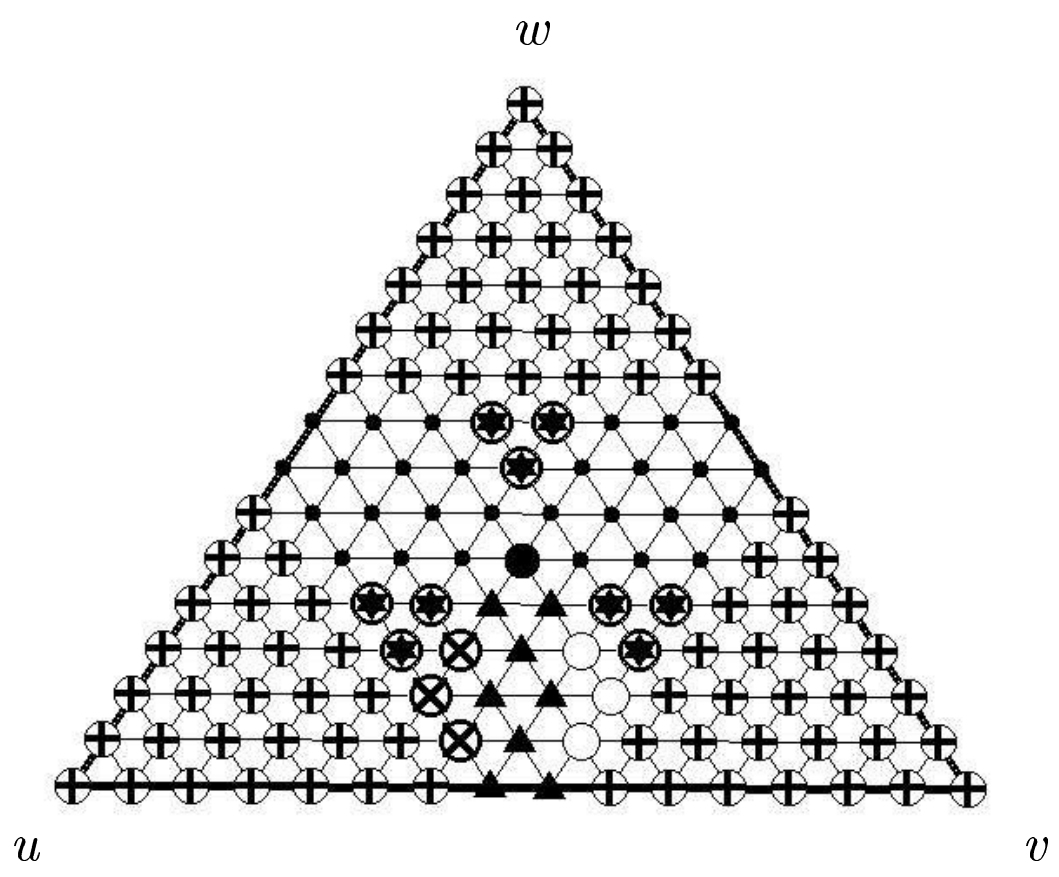

Fig. 3. Domain points for $r=4, \mu=6, d=15$.

with similar definitions for the other two vertices of $T$. Associated with the edge $e:=\langle u, v\rangle$, let

$$
\begin{aligned}
F^{T}(e) & :=\left\{\xi_{i j k}^{T}: k \leq r\right\} \\
G_{L}^{T}(e) & :=\bigcup_{i=1}^{\left\lfloor\frac{r}{2}\right\rfloor} \bigcup_{j=0}^{i-1}\left\{\xi_{d-2 r+i-1, r+1+j, r-i-j}^{T}\right\} \\
G_{R}^{T}(e) & :=\bigcup_{i=1}^{\left\lfloor\frac{r}{2}\right\rfloor} \bigcup_{j=0}^{i-1}\left\{\xi_{r+1+j, d-2 r+i-1, r-i-j}^{T}\right\} \\
E^{T}(e) & :=F^{T}(e) \backslash\left[D_{\mu}^{T}(u) \cup D_{\mu}^{T}(v) \cup A^{T}(u) \cup A^{T}(v) \cup G_{L}^{T}(e) \cup G_{R}^{T}(e)\right],
\end{aligned}
$$

with similar definitions for the other two edges of $T$. Note that $G_{L}^{T}(\langle u, v\rangle)=$ $G_{R}^{T}(\langle v, u\rangle)$.

As an aid to visualizing where these points are located, in Fig. 3 we show them for the case where $r=4, \mu=6$, and $d=15$. The points in the three disks $D_{6}(u)$, $D_{6}(v)$, and $D_{6}(w)$ are marked with the symbol $\oplus$. The points in the three sets $A^{T}(u), A^{T}(v)$ and $A^{T}(w)$ are marked with stars, and the point in $C^{T}$ is marked with a dark circle. The points in $E^{T}(e)$ for edge $e:=\langle u, v\rangle$ are marked with triangles, and the points in $G_{L}^{T}(e)$ and $G_{R}^{T}(e)$ are marked with the symbols $\otimes$ and and open circle, respectively. For clarity, we have not marked points associated with the other two edges. 
Theorem 9.1. Let $\mathcal{M}$ be the following set of domain points:

1) for each triangle $T$, include the set $C^{T}$,

2) for each edge $e$, include the set $E^{T}(e)$, where $T$ is some triangle sharing $e$,

3) for each edge of a triangle $T$ such that $e$ lies on the boundary of $\Omega$, include the sets $G_{L}^{T}(e)$ and $G_{R}^{T}(e)$,

4) for each vertex $v \in \mathcal{V}$, include $D_{\mu}^{T}(v)$ for some triangle $T$ attached to $v$,

5) suppose the vertex $v \notin \mathcal{V}_{N S}$ is connected to $v_{1}, \ldots, v_{n}$ in counterclockwise order. Let $T_{i}:=\left\langle v, v_{i}, v_{i+1}\right\rangle$ and set $T_{0}:=T_{n}=\left\langle v, v_{n}, v_{1}\right\rangle$ if $v$ is an interior vertex. Let $1 \leq i_{1}<\cdots<i_{k}<n$ be such that $e_{i_{j}}$ is $\theta_{\triangle}$-near-degenerate at either end, where $e_{i}:=\left\langle v, v_{i}\right\rangle$ for $i=1, \ldots, n$. Let $J_{v}:=\left\{i_{1}, \ldots, i_{k}\right\}$. Then

a) include $G_{L}^{T_{i}}\left(e_{i}\right)$ for all $i \in J_{v}$,

b) include $A^{T_{i}}(v)$ for all $1 \leq i \leq n-1$ such that $i \notin J_{v}$,

c) include $A^{T_{n}}(v)$ if $v$ is an interior vertex,

6) for each vertex $v \in \mathcal{V}_{S}$, include the sets $\mathcal{M}_{v, \mu+1}, \ldots, \mathcal{M}_{v, 2 r}$ constructed in Theorem 6.1,

7) for each $v \in \mathcal{V}_{N S} \backslash \mathcal{V}_{S}$ include the sets $\mathcal{M}_{v, \mu+1}, \ldots, \mathcal{M}_{v, 2 r}$ constructed in Theorem 6.2.

Then $\mathcal{M}$ is a stable local minimal determining set for $\mathcal{S}_{d}^{r, \mu}(\triangle)$.

Proof: We claim that $\mathcal{M}$ is well defined. In particular, if $v \notin \mathcal{V}_{N S}$, then by Lemma 8.2 there exists at least one edge attached to $v$ which is not $\theta_{\triangle}$-neardegenerate at either end. In the numbering of the edges in item 5) above, we can choose this edge to be $\left\langle v, v_{n}\right\rangle$, and the construction insures that for each interior vertex $v \notin \mathcal{V}_{N S}$ and edge $e_{i}:=\left\langle v, v_{i}\right\rangle$ attached to it, if $v_{i} \notin \mathcal{V}_{N S}$, then $\mathcal{M}$ includes exactly one of the two sets $A^{T_{i}}(v)$ or $G_{L}^{T_{i}}\left(e_{i}\right)$. The construction also guarantees that for all vertices $v \notin \mathcal{V}_{N S}$, there is at least one triangle $T$ with vertex at $v$ such that $\mathcal{M}$ contains the set $A^{T}(v)$.

To see that $\mathcal{M}$ is a determining set for $\mathcal{S}_{d}^{r, \mu}(\triangle)$, we show that setting $c_{\xi}=0$ for all $\xi \in \mathcal{M}$ implies $s$ is identically zero. Since for every vertex $v$ of $\triangle$ the set $\mathcal{M}$ contains $D_{\mu}^{T}(v)$ for some triangle attached to $v$, using the smoothness conditions and Lemma 4.1 we immediately see that all coefficients of $s$ associated with domain points in the disks $D_{\mu}(v)$ vanish.

Next we compute coefficients on the rings $R_{\mu+1}(v)$ for all $v$. First we do the vertices $v$ which are not in $\mathcal{V}_{N S}$. As in [25], we process arcs in a counterclockwise direction around $v$, starting with an edge $e$ such that the preceeding triangle $T$ contains the set $A^{T}(v)$. These computations are based on the smoothness conditions of Lemma 4.1, or (only if the corresponding edge is not $\theta_{\triangle}$-near-degenerate) those of Lemma 4.2. Next we use Theorem 6.1 for each vertex $v \in \mathcal{V}_{S}$, and Theorem 6.2 for each vertex in $\mathcal{V}_{N S} \backslash \mathcal{V}_{S}$. To do this, we need the coefficients corresponding to the sets $O_{v, \mu+1}$, but these will all have been set to zero or computed at this point. We now repeat this entire process one ring at a time until we have completed all of the rings up to $R_{2 r}(v)$ for all $v$. 
At this point we have shown that all coefficients of $s$ corresponding to domain points in the disks $D_{2 r}(v)$ are zero. Since $\mathcal{M}$ contains the sets $C^{T}$, the only coefficients remaining correspond to points in sets of the form

$$
E^{T}(e) \backslash\left[D_{2 r}(v) \cup D_{2 r}(u)\right],
$$

where $e=\langle v, u\rangle$ is an interior edge. These coefficients can be computed from the associated coefficients in the neighboring triangle (which will have been set to zero) using smoothness conditions as in Lemma 4.1.

We have shown that $\mathcal{M}$ is a determining set for $\mathcal{S}_{d}^{r}(\triangle)$. To see that it is minimal, we simply check that its cardinality is equal to the dimension of $\mathcal{S}_{d}^{r}(\triangle)$ as given in (2.9) of [24]. Let

$$
\begin{aligned}
& n_{a}:=\# A^{T}(v)=\# G_{L}^{T}(e)=\# G_{R}^{T}(e)=\left(\begin{array}{c}
2 r-\mu+1 \\
2
\end{array}\right), \\
& n_{c}:=\# C^{T}=\left(\begin{array}{c}
d-3 r-1 \\
2
\end{array}\right) \\
& n_{d}:=\# D_{\mu}^{T}(v)=\left(\begin{array}{c}
\mu+2 \\
2
\end{array}\right), \\
& n_{e}:=\# E^{T}(e)=n_{f}-4 n_{a}, \\
& n_{f}:=\frac{(r+1)(2 d-4 \mu+r-2)}{2} .
\end{aligned}
$$

It is easy to check that the number of points chosen in item 6 ) is $5 n_{a}$ and in item 7) is $4 n_{a}$. This is $n_{a}$ points for each edge attached to $v$, and an additional $n_{a}$ points when $v$ is singular. It follows that

$$
\# \mathcal{M}=n_{d} V+n_{a}\left(2 E+S+E_{B}\right)+n_{e} E+n_{c} N
$$

where

$$
\begin{aligned}
& E=\text { number of edges of } \triangle, \\
& E_{B}=\text { number of boundary edges of } \triangle, \\
& N=\text { number of triangles of } \triangle, \\
& S=\text { number of singular vertices of } \triangle, \\
& V=\text { number of vertices of } \triangle .
\end{aligned}
$$

Using the fact that $3 N=2 E_{I}+E_{B},(9.3)$ reduces to

$$
\# \mathcal{M}=n_{d} V+n_{a}(S-3 N)+n_{f} E+n_{c} N,
$$

which is the formula (2.9) in [24]. 
This completes the proof that $\mathcal{M}$ is a MDS for $\mathcal{S}_{d}^{r}(\triangle)$. We now claim that the corresponding dual splines $B_{\xi}$ satisfy (5.3) for some constant $K$ depending only on $\theta_{\triangle}$, i.e., $\mathcal{M}$ is stable. To see this, fix $\xi \in \mathcal{M}$, and choose $c_{\eta}$ satisfying (5.1). The remaining coefficients of $B_{\xi}$ can now be computed from smoothness conditions proceeding in the same order as described above in proving that $\mathcal{M}$ was a determining set. But the computations in Lemma 4.1 are always stable, and

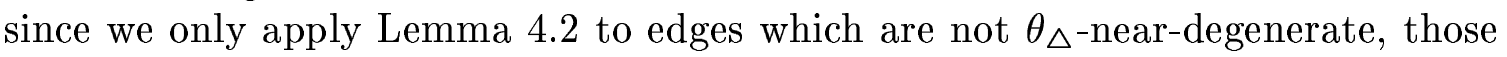
computations are also stable. The computations in the rings $R_{\mu+1}(v), \ldots, R_{2 r}(v)$ for a singular or $\theta_{\triangle}$-near-singular vertex $v$ are stable by Theorems 6.1 and 6.2.

Finally, we claim that for each $\xi \in \mathcal{M}$, the support of the dual spline $B_{\xi}$ is at most $\operatorname{star}^{3}\left(v_{\xi}\right)$ for some vertex $v_{\xi}$ depending on $\xi$. We divide the discussion into four cases. Let $\mathcal{M}_{0}:=\mathcal{M} \backslash\{\xi\}$.

Case 1: Suppose $\xi \in C^{T}$, where $T:=\left\langle v_{1}, v_{2}, v_{3}\right\rangle$ with edges $e_{i}:=\left\langle v_{i}, v_{i+1}\right\rangle$. Then by Lemma 9.2 below, the coefficients of $B_{\xi}$ must be zero on all three disks $D_{2 r}\left(v_{i}\right)$. But since $E^{T}\left(e_{i}\right) \backslash\left[D_{2 r}\left(v_{i}\right) \cup D_{2 r}\left(v_{i+1}\right)\right] \subseteq \mathcal{M}_{0}$, the coefficients of $B_{\xi}$ are also zero on these sets, and we conclude that the support of $B_{\xi}$ is just the triangle $T$.

Case 2: Suppose $\xi \in E^{T}\left(e_{1}\right) \backslash\left[D_{2 r}\left(v_{1}\right) \cup D_{2 r}\left(v_{2}\right)\right]$, where $T:=\left\langle v_{1}, v_{2}, v_{3}\right\rangle$ and $e_{1}:=\left\langle v_{1}, v_{2}\right\rangle$ is a boundary edge of $\triangle$. In this case $B_{\xi}$ has zero coefficients on the disks $D_{2 r}\left(v_{i}\right)$ and on $E^{T}\left(e_{j}\right) \backslash\left[D_{2 r}\left(v_{j}\right) \cup D_{2 r}\left(v_{j+1}\right)\right]$ for the other two edges $e_{j}:=\left\langle v_{j}, v_{j+1}\right\rangle$ of $T$. It follows that the support of $B_{\xi}$ is just the triangle $T$.

Case 3: Suppose $\xi \in E^{T}(e) \backslash\left[D_{2 r}(v) \cup D_{2 r}(u)\right]$, where $e=\langle v, u\rangle$ is an interior edge shared by $T$ and a neighboring triangle $\widetilde{T}$. Then arguing as in Case 2 , we see that the support of $B_{\xi}$ is $T \cup \widetilde{T}$.

The situation is more complicated when $\xi$ lies in some disk $D_{2 r}(v)$. This is due to the fact that when $d<4 r+1$ the $2 r$-disks overlap, and nonzero coefficients in one such disk can propagate to a neighboring disk as discussed in [25].

Case 4: Suppose $\xi \in \mathcal{M} \cap D_{2 r}(v)$. Suppose $z_{1}, \ldots, z_{n}$ are the points on the boundary of $\operatorname{star}^{3}(v)$ in counterclockwise order. Then Lemma 9.2 below shows that the coefficients of $B_{\xi}$ are zero on the disks $D_{2 r}\left(z_{i}\right)$. Now for each $e_{i}:=\left\langle z_{i}, z_{i+1}\right\rangle$, $E^{T_{i}}\left(e_{i}\right) \backslash\left[D_{2 r}\left(z_{i}\right) \cup D_{2 r}\left(z_{i+1}\right)\right] \subseteq \mathcal{M}_{0}$ for some triangle $T_{i}$ sharing the edge $e_{i}$. It follows that the corresponding coefficients are also zero, and we conclude that the support of $B_{\xi}$ is a subset of $\operatorname{star}^{3}(v)$.

The following lemma is used in the proof of the previous theorem.

Lemma 9.2. Let $\mathcal{M}$ be the $M D S$ in Theorem 9.1, and let $B_{\xi}$ be the dual spline corresponding to a $\xi \in \mathcal{M}$. Then $B_{\xi}$ can have a nonzero coefficient corresponding to a domain point in a disk $D_{2 r}(w)$ only if $\xi \in D_{2 r}(v)$ for some $v$, and either $v=w$, or $w$ is connected directly to $v$ with an edge $\langle w, v\rangle$ or by a pair of edges $\langle w, u\rangle$, $\langle u, v\rangle$, where $u$ is a $\theta_{\triangle}$-near-singular vertex.

Proof: It is clear from the first part of the proof of Theorem 9.1 that the coefficients in a disk $D_{2 r}(w)$ are computed from smoothness conditions which involve only 
coefficients in such disks. Hence, if $\xi$ is not in any disk $D_{2 r}(v)$, then $B_{\xi}$ has zero coefficients on all disks $D_{2 r}(w)$.

Suppose now that $\xi \in D_{2 r}(v)$ and that $w \neq v$ is not connected directly to $v$ with an edge $\langle w, v\rangle$ or by a pair of edges $\langle w, u\rangle,\langle u, v\rangle$ where $u$ is a $\theta_{\triangle}$-near-singular vertex. Let $w_{1}, \ldots, w_{n}$ be the vertices attached to $w$ and let $e_{i}:=\left\langle w, w_{i}\right\rangle$ and $T_{i}:=\left\langle w, w_{i}, w_{i+1}\right\rangle$. Clearly, $D_{\mu}(w) \subseteq \mathcal{M}_{0}$ and $E\left(e_{i}\right) \subseteq \mathcal{M}_{0}$ for each $1 \leq i \leq n$, where $E\left(e_{i}\right)$ is one of the sets $E^{T_{i}}\left(e_{i}\right)$ and $E^{T_{i-1}}\left(e_{i}\right)$. There are two cases.

Case 1: $w \in \mathcal{V}_{N S}$. In this case the four vertices $w_{1}, \ldots, w_{4}$ are all different from $v$, which insures $D_{\mu}\left(w_{i}\right) \subseteq \mathcal{M}_{0}$ for $i=1, \ldots, 4$. Moreover, none of the $w_{i}$ is $\theta_{\triangle}$-nearsingular since two $\theta_{\triangle}$-near-singular vertices cannot be neighbors. Since the edges $\left\langle w, w_{i}\right\rangle$ are all $\theta_{\triangle}$-near-degenerate at $w$, we conclude that $\mathcal{M}_{0}$ also contains the sets $G_{R}^{T_{i-1}}\left(e_{i}\right), 1 \leq i \leq 4$. This implies that all coefficients of $B_{\xi}$ in $D_{2 r}(w)$ must be zero. To see this, we first calculate the coefficients on the ring $R_{\mu+1}(w)$ from the nonsingular system (6.10) in the proof of Theorem 6.2. We will get zero coefficients if the right-hand side is zero, which happens as soon as the coefficients associated with domain points in $D_{\mu}^{T_{1}}(w)$ and the sets $\mathcal{M}_{w, \mu+1}$ and $O_{w, \mu+1}$ in the theorem are zero. Since $D_{\mu}^{T_{1}}(w) \cup \mathcal{M}_{w, \mu+1} \subseteq \mathcal{M}_{0}$, we only have to check $O_{w, \mu+1}$. It is easy to see that $O_{w, \mu+1} \subseteq \bigcup_{i=1}^{4} E\left(e_{i}\right)$, and it follows that the coefficients in the disk $D_{\mu+1}(w)$ are zero. Repeating this process for each of the rings $R_{\mu+2}(w), \ldots, R_{2 r}(w)$, we note that for each $k=\mu+2, \ldots, 2 r$,

$$
O_{w, k} \subseteq \bigcup_{i=1}^{4}\left[D_{\mu}\left(w_{i}\right) \cup G_{R}^{T_{i-1}}\left(e_{i}\right) \cup G_{R}^{T_{i}}\left(e_{i}\right) \cup E^{T_{i-1}}\left(e_{i}\right) \cup E^{T_{i}}\left(e_{i}\right)\right]
$$

Since the coefficients corresponding to $\mathcal{M}_{0}$ are zero, and the coefficients corresponding to the disk $D_{k-1}(w)$ are also zero by the induction hypothesis, it is easy to see that the coefficients associated with points in $O_{w, k}$ must be zero, which in turn implies that we have only zero coefficients associated with $R_{k}(w)$.

Case 2: $w \notin \mathcal{V}_{N S}$. By Lemma 8.2, there is at least one edge attached to $w$ which is not $\theta_{\triangle}$-near-degenerate at either end. Without loss of generality we may assume it is $e_{n}$. Then $A^{T_{n}}(w) \subseteq \mathcal{M}_{0}$, and the corresponding coefficients of $B_{\xi}$ must be zero. We now compute coefficients on the ring $R_{\mu+1}(w)$ proceeding in counterclockwise order around $w$. For $i=1, \ldots, n$, we show that coefficients on the arc $a_{\mu+1, e_{i}}^{r}(w)$ are all zero. Assuming this holds for for all $i \leq k-1$, we now show it for $i=k$.

a) If $e_{k}$ is not $\theta_{\triangle}$-near-degenerate at either end, then $\mathcal{M}_{0}$ contains $A^{T_{k}}(w)$ and either $E^{T_{k}}\left(e_{k}\right)$ or $E^{T_{k-1}}\left(e_{k}\right)$. Hence the coefficients for points in $a_{\mu+1, e_{k}}^{r}(w) \backslash$ $\left(G_{L}^{T_{k-1}}\left(e_{k}\right) \cup G_{L}^{T_{k}}\left(e_{k}\right)\right)$ are zero, and we get all zero coefficients for points on the arc $a_{\mu+1, e_{k}}^{r}(w)$ by Lemma 4.2.

b) If $e_{k}$ is $\theta_{\triangle}$-near-degenerate at one end and $w_{k} \notin \mathcal{V}_{N S}$, then $\mathcal{M}_{0}$ contains all of the sets $D_{\mu}(w), D_{\mu}\left(w_{k}\right), G_{R}^{T_{k-1}}\left(e_{k}\right), G_{L}^{T_{k}}\left(e_{k}\right)$ and either $E^{T_{k}}\left(e_{i}\right)$ or $E^{T_{k-1}}\left(e_{i}\right)$. Moreover, by the induction hypothesis, the coefficients for points in 


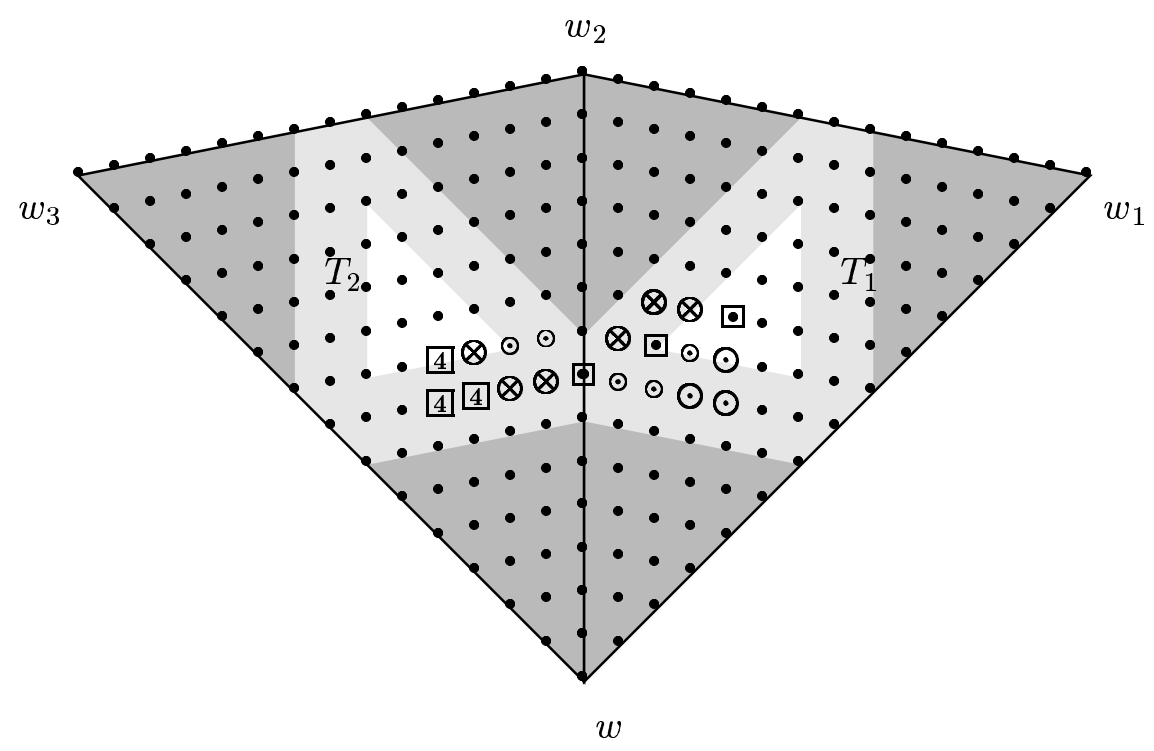

Fig. 4. Blocking propagation.

$a_{\mu+1, e_{k}}^{r}(w) \cap a_{\mu+1, e_{k-1}}^{r}(w)$ are zero, too. Then using the smoothness condition, it follows that all coefficients associated with points on the $\operatorname{arc} a_{\mu+1, e_{k}}^{r}(w)$ must be zero (cf. Lemma 8.2 of [25]).

c) If $w_{k} \in \mathcal{V}_{N S}$, then $w_{k}$ is not connected directly to $v$ with an edge $\left\langle w_{k}, v\right\rangle$ or by a pair of edges $\left\langle w_{k}, u\right\rangle,\langle u, v\rangle$ with $u$ a $\theta_{\triangle}$-near-singular vertex. Then, by Case 1 , all coefficients of $B_{\xi}$ associated with points in $D_{2 r}\left(w_{k}\right)$ must be zero, and the same argument as in b) shows that all coefficients associated with points on the $\operatorname{arc} a_{\mu+1, e_{k}}^{r}(w)$ must be zero.

To complete the proof, we now repeat this process for each of the rings $R_{\mu+2}(w), \ldots, R_{2 r}(w)$.

Fig. 4 illustrates Case 2b of Lemma 9.2 for $r=4, \mu=6$, and $d=14$. Suppose $e_{2}$ is $\theta_{\triangle}$-near-degenerate at either $w_{2}$ or $w$. Then the coefficients corresponding to points in the sets $G_{L}^{T_{2}}\left(e_{2}\right)$ and $G_{R}^{T_{1}}\left(e_{2}\right)$ are zero. We have marked those points with the symbol $\otimes$. The coefficients associated with the points in $E^{T}\left(e_{2}\right)$ and $A^{T_{1}}(w)$ are also zero. They are marked with $\square$ and $\odot$, respectively. Then using smoothness conditions, we see that all of the coefficients corresponding to points marked with $\odot$ along with those in $A^{T_{2}}(w)$ (marked with the number 4 ) must be zero.

We conclude this section with an example to illustrate that propagation to $\operatorname{star}^{3}(v)$ can actually happen.

Example 9.3. Let $\triangle$ be the triangulation shown in Fig. 5, and let $r=5, \mu=8$, $d=17$.

Discussion: For ease of understanding, we shade the disks $D_{\mu}$ and $D_{2 r}$ in dark and light gray, respectively. Suppose $\mathcal{M}$ contains the set $D_{\mu}^{T}(v)$ where $T$ is the 


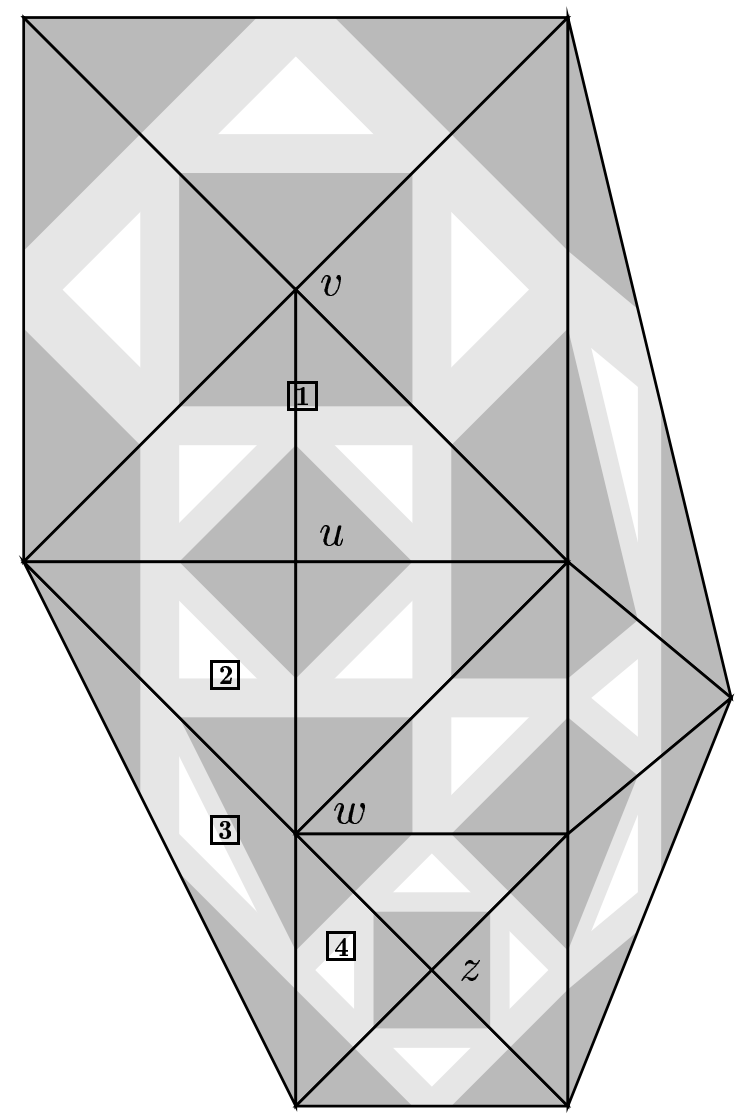

Fig. 5. Propagation to $\operatorname{star}^{3}(v)$.

top triangle in the figure, and suppose $\xi$ is the point at the vertex $v$. Then $B_{\xi}$ has support on all of the triangles surrounding $v$, and in particular, it has a nonzero coefficient corresponding to the point $R_{\mu}(v) \cap\langle v, u\rangle$. This point is numbered 1 in the figure, and can be identified with the point marked with a $\oplus$ in Fig. 2. As seen from that figure, the nonzero coefficient at point number 1 can propagate to a nonzero coefficient corresponding to the point in the set $A^{T_{1}}(w)$ which is marked with a $\otimes$ in Fig. 2 and with the number 2 here. (We assume $w_{1}=u$ and $w_{4}=z$.) Assuming both $\left\langle w, w_{2}\right\rangle$ and $\left\langle w, w_{3}\right\rangle$ are $\theta_{\triangle}$-near-degenerate, we get further propagation to a point in the set $A^{T_{3}}(w)$ marked with the number 4 . This set lies in $\operatorname{star}^{3}(v)$ but outside of $\operatorname{star}^{2}(v)$.

\section{$\S 10$. A stable basis for $\mathcal{S}_{\mu}^{r, \rho_{v}}\left(\triangle_{v}\right)$ on a cell $\triangle_{v}$}

Before constructing stable local bases for $\mathcal{S}_{d}^{r}(\triangle)$ and for general superspline spaces, we need to examine the superspline space $\mathcal{S}_{\mu}^{r, \rho_{v}}\left(\triangle_{v}\right)$ with $r \leq \rho_{v}<\mu$ on an arbitrary 
cell $\triangle_{v}$. Suppose that $v$ is a vertex which is connected to the vertices $v_{1}, \ldots, v_{n}$ in counterclockwise order, and let $v_{n+1}=v_{1}$. Let

$$
\triangle_{v}:=\left\{T_{i}:=\left\langle v, v_{i}, v_{i+1}\right\rangle, \quad i=1, \ldots, n\right\}
$$

form a triangulation of the set

$$
\Omega_{v}:=\bigcup_{i=1}^{n} T_{i}
$$

In this case $\triangle_{v}$ is called an interior cell. We now construct a stable basis for $\mathcal{S}_{\mu}^{r, \rho_{v}}\left(\triangle_{v}\right)$.

Let $e$ be the number of edges attached to $v$ with different slopes. Then by Theorem 2.2 of [30],

$$
m:=\operatorname{dim} \mathcal{S}_{\mu}^{r, \rho_{v}}\left(\triangle_{v}\right)=\left(\begin{array}{c}
\rho_{v}+2 \\
2
\end{array}\right)+n\left[\left(\begin{array}{c}
\mu-r+1 \\
2
\end{array}\right)-\left(\begin{array}{c}
\rho_{v}-r+1 \\
2
\end{array}\right)\right]+\sigma
$$

where

$$
\sigma:=\sum_{j=\rho_{v}-r+1}^{\mu-r}(r+j+1-j e)_{+} .
$$

Suppose $\left\{\xi_{i}\right\}_{i=1}^{n_{c}}$ are the domain points associated with the cell $\triangle_{v}$. It is easy to see that

$$
n_{c}=n\left[\left(\begin{array}{c}
\mu-1 \\
2
\end{array}\right)+2 \mu-1\right]+1=n\left[\frac{\mu^{2}+\mu}{2}\right]+1 .
$$

Given $s \in \mathcal{S}_{\mu}^{r, \rho_{v}}\left(\triangle_{v}\right)$, we denote the B-coefficient associated with $\xi_{i}$ by $c_{i}$ for $i=$ $1, \ldots, n_{c}$. Associated with each interior edge of $\triangle_{v}$, there are $\mu-j+1$ smoothness conditions to insure $C^{j}$ continuity across that edge, $j=1, \ldots, r$, and $\rho-r-k+1$ smoothness conditions to insure $C^{\rho_{v}}$ continuity at $v, k=1, \ldots, \rho_{v}-r$. This gives a total of

$$
\begin{aligned}
n_{s} & :=n\left[\left(\begin{array}{c}
\mu+1 \\
2
\end{array}\right)-\left(\begin{array}{c}
\mu-r+1 \\
2
\end{array}\right)+\left(\begin{array}{c}
\rho_{v}-r+1 \\
2
\end{array}\right)\right] \\
& =n r\left[\frac{2 \mu-r+1}{2}\right]+n\left(\begin{array}{c}
\rho_{v}-r+1 \\
2
\end{array}\right)
\end{aligned}
$$

smoothness conditions to insure that $s$ lies in $\mathcal{S}_{\mu}^{r, \rho_{v}}\left(\triangle_{v}\right)$. Note that $n_{s}<n_{c}$. These conditions can be written in matrix form

$$
A c=0
$$

where $c=\left(c_{1}, \ldots, c_{n_{c}}\right)^{T}$, and $A$ is an appropriate $n_{s} \times n_{c}$ matrix. 
In general, the system (10.5) includes some redundant smoothness conditions, and so $n_{r}:=\operatorname{rank}(A)<n_{s}$. Indeed, since $\operatorname{dim} \mathcal{S}_{\mu}^{r, \rho_{v}}\left(\triangle_{v}\right)=n_{c}-n_{r}$, it follows that

$$
\begin{aligned}
n_{r} & =n\left[\frac{\mu^{2}+\mu}{2}\right]+1-\left(\begin{array}{c}
\rho_{v}+2 \\
2
\end{array}\right)-n\left[\left(\begin{array}{c}
\mu-r+1 \\
2
\end{array}\right)-\left(\begin{array}{c}
\rho_{v}-r+1 \\
2
\end{array}\right)\right]-\sigma \\
& =n r\left[\frac{2 \mu-r+1}{2}\right]+1-\left(\begin{array}{c}
\rho_{v}+2 \\
2
\end{array}\right)+n\left(\begin{array}{c}
\rho_{v}-r+1 \\
2
\end{array}\right)-\sigma .
\end{aligned}
$$

This implies that the number of redundant equations in (10.5) is

$$
n_{r e d}:=\left(\begin{array}{c}
\rho_{v}+2 \\
2
\end{array}\right)-1+\sigma
$$

Without loss of generality, we may assume that redundant equations have been dropped, and that (10.5) is written in the equivalent form

$$
\left[A_{1} A_{2}\right] c=0
$$

where $A_{1}$ is an $n_{r} \times m$ matrix and $A_{2}$ is an $n_{r} \times n_{r}$ matrix. We may also assume that the columns of $A$ (and the corresponding components of $c$ ) have been numbered so that the determinant of $A_{2}$ has the maximal absolute value over all $n_{r} \times n_{r}$ subdeterminants of $A$.

Algorithm 10.1. For each $i=1, \ldots, m$, let $s_{i}$ be the spline in $\mathcal{S}_{\mu}^{r, \rho_{v}}\left(\triangle_{v}\right)$ with $B$-coefficients $c=\left(c_{1}, \ldots, c_{n_{c}}\right)^{T}$ chosen so that $c_{i}=1, c_{j}=0$ for $j=1, \ldots, m$ with $j \neq i$, and $c_{m+1}, \ldots, c_{n_{c}}$ are determined from the linear system

$$
A_{2}\left(\begin{array}{c}
c_{m+1} \\
\vdots \\
c_{n_{c}}
\end{array}\right)=-A_{1}(i)
$$

where $A_{1}(i)$ is the $i$-th column of the matrix $A_{1}$.

The splines $\left\{s_{i}\right\}_{i=1}^{m}$ are clearly linearly independent since

$$
\lambda_{j} s_{i}=\delta_{i, j}, \quad j=1, \ldots, m,
$$

where $\lambda_{j}$ is the linear functional which picks off the $j$-th B-coefficient. It follows that they form a basis for $\mathcal{S}_{\mu}^{r, \rho_{v}}\left(\triangle_{v}\right)$. We now show that their construction is a stable process, i.e., for each $i$, all of the coefficients of $s_{i}$ are uniformly bounded.

Theorem 10.2. Suppose $s_{i}$ is a basis spline constructed by Algorithm 10.1. Then its B-coefficients satisfy

$$
\left|c_{j}\right| \leq 1, \quad j=1, \ldots, n_{c} .
$$


Proof: Fix $1 \leq i \leq m$, and let $c=\left(c_{1}, \ldots, c_{n_{c}}\right)$ be the vector of coefficients of $s_{i}$ as computed from Algorithm 10.1. Then (10.10) clearly holds for $j=1, \ldots, m$. Let $m+1 \leq j \leq n_{c}$. Then by Cramer's rule,

$$
c_{j}=\frac{\operatorname{det}\left(\tilde{A}_{2}\right)}{\operatorname{det}\left(A_{2}\right)},
$$

where $\tilde{A}_{2}$ is the matrix obtained from $A_{2}$ by replacing the $j$-th column by $-A_{1}(i)$. But then $\left|c_{j}\right| \leq 1$ follows by the choice of $A_{2}$.

Note that this is a constructive algorithm for building dual basis splines. Indeed, if we take $\mathcal{M}_{v}$ to be the set of domain points corresponding to the $m$ coefficients $c_{1}, \ldots, c_{m}$ which are set (as opposed to calculated) in Algorithm 10.1, then obviously $\mathcal{M}_{v}$ is a minimal determining set for $\mathcal{S}_{\mu}^{r, \rho_{v}}\left(\triangle_{v}\right)$.

A completely analogous algorithm can be used to create stable dual basis splines for $\mathcal{S}_{\mu}^{r, \rho_{v}}\left(\triangle_{v}\right)$ in the case where $\triangle_{v}$ is a boundary cell.

\section{$\S 11$. A stable basis for $\mathcal{S}_{d}^{r, \rho}(\triangle)$}

In this section we combine the constructions of the two previous sections to create stable local bases for the spaces of supersplines $\mathcal{S}_{d}^{r, \rho}(\triangle)$ defined in (1.1) for all $d \geq 3 r+2$. As in [24], we assume that

$$
k_{v}+k_{u}<d \text { for each pair of neighboring vertices } v, u \in \mathcal{V} \text {, }
$$

where

$$
k_{v}:=\max \left\{\rho_{v}, \mu\right\}, \quad v \in \mathcal{V},
$$

with $\mu$ as in (1.3).

Given a triangle $T=\langle u, v, w\rangle$, let

$$
\tilde{C}^{T}:=C^{T} \backslash\left[D_{k_{u}}^{T}(u) \cup D_{k_{v}}^{T}(v) \cup D_{k_{w}}^{T}(w)\right] .
$$

Associated with $u$, let

$$
\tilde{A}^{T}(u):=A^{T}(u) \backslash D_{k_{u}}^{T}(u),
$$

with similar definitions for the other two vertices of $T$. Associated with the edge $e:=\langle u, v\rangle$, we define

$$
\begin{aligned}
\tilde{G}_{L}^{T}(e) & :=G_{L}^{T}(e) \backslash D_{k_{u}}^{T}(u) \\
\tilde{G}_{R}^{T}(e) & :=G_{R}^{T}(e) \backslash D_{k_{v}}^{T}(v), \\
\tilde{E}^{T}(e) & :=E^{T}(e) \backslash\left[D_{k_{u}}^{T}(u) \cup D_{k_{v}}^{T}(v)\right],
\end{aligned}
$$

with similar definitions for the other edges of $T$. 
Theorem 11.1. Let $\mathcal{M}$ be the following set of domain points:

1) for each triangle $T$, include the set $\tilde{C}^{T}$,

2) for each edge $e$, include the set $\tilde{E}^{T}(e)$, where $T$ is some triangle sharing $e$,

3) for each edge of a triangle $T$ such that $e$ lies on the boundary of $\Omega$, include the sets $\tilde{G}_{L}^{T}(e)$ and $\tilde{G}_{R}^{T}(e)$,

4) for each vertex $v \in \mathcal{V}$,

a) include the set $D_{\rho_{v}}^{T}(v)$ for some triangle attached to $v$ if $\rho_{v} \geq \mu$,

b) include the domain points in $D_{\mu}(v)$ corresponding to the stable minimal determining set $\mathcal{M}_{v}$ of Section 10 for $S_{\mu}^{r, \rho_{v}}\left(\triangle_{v}\right)$ if $\rho_{v}<\mu$,

5) suppose the vertex $v \notin \mathcal{V}_{N S}$ is connected to $v_{1}, \ldots, v_{n}$ in counterclockwise order. Let $T_{i}:=\left\langle v, v_{i}, v_{i+1}\right\rangle$ and set $T_{0}:=T_{n}=\left\langle v, v_{n}, v_{1}\right\rangle$ if $v$ is an interior vertex. Let $1 \leq i_{1}<\cdots<i_{k}<n$ be such that $e_{i_{j}}$ is $\theta_{\triangle}$-near-degenerate at either end, where $e_{i}:=\left\langle v, v_{i}\right\rangle$ for $i=1, \ldots, n$. Let $J_{v}:=\left\{i_{1}, \ldots, i_{k}\right\}$. Then

a) include $\tilde{G}_{L}^{T_{i}}\left(e_{i}\right)$ for all $i \in J_{v}$,

b) include $\tilde{A}^{T_{i}}(v)$ for all $1 \leq i \leq n-1$ such that $i \notin J_{v}$,

c) include $\tilde{A}^{T_{n}}(v)$ if $v$ is an interior vertex,

6) for each vertex $v \in \mathcal{V}_{S}$, include the sets $\mathcal{M}_{v, k_{v}+1}, \ldots, \mathcal{M}_{v, 2 r}$ constructed in Theorem 6.1,

7) for each $v \in \mathcal{V}_{N S} \backslash \mathcal{V}_{S}$ include the sets $\mathcal{M}_{v, k_{v}+1}, \ldots, \mathcal{M}_{v, 2 r}$ constructed in Theorem 6.2.

Then $\mathcal{M}$ is a stable local minimal determining set for $\mathcal{S}_{d}^{r, \rho}(\triangle)$.

Proof: It is straightforward to check that $\mathcal{M}$ is a determining set for $\mathcal{S}_{d}^{r, \rho}(\triangle)$. To see that it is minimal, we check that its cardinality is equal to the formula $(2.16)$ in [24] for the dimension of $\mathcal{S}_{d}^{r, \rho}(\triangle)$. Then locally supported dual basis splines $B_{\xi}$ can be stably constructed in the same way as in the proof of Theorem 9.1.

\section{$\S 12$. Stability and local linear independence}

We recall (cf. $[13,16,17,19,20,21])$ that a set $\mathcal{B}=\left\{B_{\nu}\right\}_{\nu \in \mathcal{I}}$ of basis splines for a spline space $\mathcal{S}$ is called locally linearly independent (LLI) provided that for every $T \in \triangle$, the splines $\left\{B_{\nu}\right\}_{\nu \in \Sigma_{T}}$ are linearly independent on $T$, where

$$
\Sigma_{T}:=\left\{\nu: T \subseteq \operatorname{supp} B_{\nu}\right\} .
$$

Since the classical univariate $B$-splines are both stable and locally linearly independent (cf. Theorems 4.18 and 4.41 in [29]), it seems natural to expect that there also exist bases for bivariate spline spaces which possess both of these properties simultaneously. Here we have constructed stable local bases for the spline spaces $\mathcal{S}_{d}^{r}(\triangle)$ and their superspline subspaces, while star-supported LLI bases for the same spaces were recently constructed in [19]. But these bases are different, and in fact we have the following surprising result. 
Theorem 12.1. Given $r \geq 1$ and $d \geq 3 r+2$, there are triangulations such that no basis for $\mathcal{S}_{d}^{r}(\triangle)$ is simultaneously stable and locally linearly independent.

Proof: Suppose $\mathcal{B}:=\left\{B_{\nu}\right\}_{\nu \in \mathcal{I}}$ is a stable LLI basis for $\mathcal{S}_{d}^{r}(\triangle)$ on a triangulation which contains an interior near-singular vertex $v$. Following the notation of Sect. 6 , suppose $v$ is connected to $v_{1}, v_{2}, v_{3}, v_{4}$ in counterclockwise order. For each $1 \leq i \leq 4$, let $e_{i}:=\left\langle v, v_{i}\right\rangle$, and $T_{i}:=\left\langle v, v_{i}, v_{i+1}\right\rangle$. Suppose

$$
v_{i-1}=r_{i} v_{i+1}+s_{i} v+t_{i} v_{i}
$$

and suppose that none of the $e_{i}$ is degenerate at $v$, i.e., $t_{i} \neq 0$. For convenience, we define $\alpha_{i}, \beta_{i}, \gamma_{i}, \mu_{i}$ to be the linear functionals picking off B-coefficients corresponding to the domain points $\xi_{d-2 r, r, r}^{T_{i}}, \xi_{d-2 r, r-1, r+1}^{T_{i}}, \xi_{d-2 r, r+1, r-1}^{T_{i}}, \xi_{d-2 r-1, r, r+1}^{T_{i}}$, respectively.

For each $1 \leq j \leq 4$, we claim that there is a unique spline $S_{j} \in \mathcal{S}_{d}^{r}(\triangle)$ whose only nonzero coefficients are

$$
\begin{gathered}
\alpha_{j} S_{j}:=1, \quad \gamma_{j} S_{j}:=-r_{j} /\left(r t_{j}\right), \quad \gamma_{j+1} S_{j}:=r_{j+1}^{1-r} /\left(r t_{j+1}\right) \\
\beta_{j-1} S_{j}:=r_{j}^{r-1} \gamma_{j} S_{j}, \quad \beta_{j} S_{j}:=r_{j+1}^{r-1} \gamma_{j+1} S_{j} \\
\mu_{j-1} S_{j}:=r r_{j}^{r-1} s_{j} \gamma_{j} S_{j}, \quad \mu_{j} S_{j}:=r r_{j+1}^{r-1} s_{j+1} \gamma_{j+1} S_{j} .
\end{gathered}
$$

It can be verified directly that $S_{j}$ satisfies all $C^{r}$ smoothness conditions, and thus belongs to $\mathcal{S}_{d}^{r}(\triangle)$. It is also easy to see that

$$
\operatorname{supp} S_{j}=T_{j-1} \cup T_{j} \cup T_{j+1},
$$

and by a property of LLI bases (see $[13,21]$ ),

$$
S_{j}=\sum_{\nu \in I_{j}} c_{\nu}^{[j]} B_{\nu}
$$

where $I_{j}:=\left\{\nu: \operatorname{supp} B_{\nu} \subseteq T_{j-1} \cup T_{j} \cup T_{j+1}\right\}$ for $j=1,2,3,4$. We now define

$$
\hat{S}=r_{2}^{r} S_{1}+S_{2}+r_{3}^{-r} S_{3}+\left(r_{3} r_{4}\right)^{-r} S_{4}
$$

The definition of barycentric coordinates implies that $r_{1} r_{2} r_{3} r_{4}=1$. Using this fact, it is easy to check that all of the coefficients of $\hat{S}$ are zero except for

$$
\alpha_{1} \hat{S}:=r_{2}^{r}, \quad \alpha_{2} \hat{S}:=1, \quad \alpha_{3} \hat{S}:=r_{3}^{-r}, \quad \alpha_{4} \hat{S}:=\left(r_{3} r_{4}\right)^{-r}
$$

(For example, $\gamma_{1} \hat{S}=r_{2}^{r} \gamma_{1} S_{1}+\left(r_{3} r_{4}\right)^{-r} \gamma_{1} S_{4}=r_{1}\left(-r_{2}^{r}+\left(r_{1} r_{3} r_{4}\right)^{-r}\right) /\left(r t_{1}\right)=0$.) By (6.13) and Lemma 4.1 of [25], this immediately implies

$$
\|\hat{S}\|_{\infty} \leq K_{3}
$$


where $K_{3}$ depends only on $d$ and $\theta_{\triangle}$.

In view of (12.2), we can write

$$
\hat{S}=\sum_{\nu \in I_{1} \cup I_{2} \cup I_{3} \cup I_{4}} a_{\nu} B_{\nu}
$$

By the assumption that the basis $\mathcal{B}$ is stable, we have

$$
\|a\|_{\infty} \leq K_{1}^{-1}\|\hat{S}\|_{\infty} \leq K_{3} / K_{1}
$$

where $K_{1}$ is the constant in (1.2).

For each $\nu$, let $\tilde{B}_{\nu}=B_{\nu}-\mathcal{T}_{2 r-1} B_{\nu}$, where $\mathcal{T}_{2 r-1} B_{\nu} \in \mathcal{S}_{2 r-1}^{r}\left(\triangle_{v}\right) \subseteq \mathcal{S}_{d}^{r}\left(\triangle_{v}\right)$ is the spline constructed in Lemma 6.4 which interpolates the derivatives of $B_{\nu}$ at $v$ up to order $2 r-1$. Then the B-coefficients of $\tilde{B}_{\nu}$ corresponding to domain points in the disk $D_{2 r-1}(v)$ are zero. Moreover, since the basis $\mathcal{B}$ is stable, it follows from Lemma 6.4 that the B-coefficients of $\tilde{B}_{\nu}$ corresponding to domain points on the ring $R_{2 r}(v)$ are bounded in absolute value by a constant $K_{4}$ depending only on $d$ and $\theta_{\triangle}$.

Since all of the derivatives of $\hat{S}$ up to order $2 r-1$ at $v$ are zero, $\mathcal{T}_{2 r-1} \hat{S}=0$, and we have (on $\triangle_{v}$ )

$$
\hat{S}=\sum_{\nu \in I_{1} \cup I_{2} \cup I_{3} \cup I_{4}} a_{\nu} \tilde{B}_{\nu}
$$

Since the support of $\tilde{B}_{\nu}$ is a subset of the support of $B_{\nu}$ (on $\triangle_{v}$ ), it follows that $\alpha_{2} \tilde{B}_{\nu} \neq 0$ only if $\nu$ lies in the set

$$
\tilde{I}_{2}:=\left\{\nu: \operatorname{supp} B_{\nu}=T_{1} \cup T_{2} \cup T_{3}\right\} .
$$

This implies

$$
1=\alpha_{2} \hat{S}=\sum_{\nu \in \tilde{I}_{2}} a_{\nu} \alpha_{2} \tilde{B}_{\nu} \leq \# \tilde{I}_{2}\|a\|_{\infty} \max _{\nu \in \tilde{I}_{2}}\left|\alpha_{2} \tilde{B}_{\nu}\right|
$$

Now clearly $\# \tilde{I}_{2} \leq 3\left(\begin{array}{c}d+2 \\ 2\end{array}\right)$, and hence there exists $\nu_{0} \in \tilde{I}_{2}$ such that

$$
\left|\alpha_{2} \tilde{B}_{\nu_{0}}\right| \geq K_{5}>0
$$

where $K_{5}$ depends only on $d$ and $\theta_{\triangle}$.

Now consider the following $C^{r}$ smoothness condition across the edge $e_{2}$ :

$$
\alpha_{1} \tilde{B}_{\nu_{0}}=r_{2}^{r} \alpha_{2} \tilde{B}_{\nu_{0}}+r r_{2}^{r-1} t_{2} \gamma_{2} \tilde{B}_{\nu_{0}}+\sum_{k=1}^{r-1}\left(\begin{array}{c}
r \\
r-k-1
\end{array}\right) r_{2}^{r-k-1} t_{2}^{k+1} \eta_{2, k} \tilde{B}_{\nu_{0}}
$$

where $\eta_{2, k}$ is the linear functional which picks off the B-coefficient corresponding to $\xi_{d-2 r, r+k+1, r-k-1}^{T_{i}}$ for $k=1, \ldots, r-1$. Since $\alpha_{1} \tilde{B}_{\nu_{0}}=0$, this implies

$$
\left|\gamma_{2} \tilde{B}_{\nu_{0}}\right|+\frac{1}{r} \sum_{k=1}^{r-1}\left(\begin{array}{c}
r \\
r-k-1
\end{array}\right)\left|\frac{t_{2}}{r_{2}}\right|^{k}\left|\eta_{2, k} \tilde{B}_{\nu_{0}}\right| \geq\left|\frac{r_{2}}{t_{2}}\right| \frac{K_{5}}{r},
$$


which is unbounded as $t_{2} \rightarrow 0$. On the other hand, since the B-coefficients $\gamma_{2} \tilde{B}_{\nu_{0}}$, $\eta_{2, k} \tilde{B}_{\nu_{0}}, k=1, \ldots, r-1$, correspond to domain points on the ring $R_{2 r}(v)$, they cannot exceed $K_{4}$ in absolute value, which leads to a contradiction and completes the proof.

Note that the above proof also applies to the superspline spaces $\mathcal{S}_{d}^{r, \rho}(\triangle)$ whenever $\rho_{v}<2 r$ for some near-singular vertex $v$. On the other hand, if $d \geq 4 r+1$ and $\rho_{v} \geq 2 r$ for all vertices, then the basis constructed in [24] is both stable and LLI.

\section{$\S 13$. Remarks}

Remark 13.1. It is well known $[5-8,23,24]$ that the dimension of spline spaces and superspline spaces (when $\rho_{v}<2 r$ ) generally depends on the exact geometry of the triangulation, and in particular may change as certain near-singular vertices become singular. Thus, it may seem surprising that it is possible to construct stable bases even though the dimension is not stable. This fact was realized already in [1] for $\mathcal{S}_{2}^{1}\left(\triangle_{v}\right)$, where $\triangle_{v}$ is a four-cell associated with a near-singular vertex. The spaces considered in [14] are also examples where stable bases were constructed even though the dimensions were not stable.

Remark 13.2. For $d \geq 3 r+2$, algorithms for constructing star-supported bases for $\mathcal{S}_{d}^{r}(\triangle)$ were presented in [23], and for general superspline subspaces in [24]. The constructions there produce stable bases for $r=0$, and for $d \geq 4 r+1, \rho_{v} \geq 2 r$ for $r>0$. However, they are not generally stable if $\rho_{v}<2 r$, since some of the basis functions do not remain bounded for sequences of triangulations containing vertices which become singular, even if the smallest angle in the triangulations is bounded away from zero. If $\rho_{v}<\mu$, then many other sequences of triangulations lead to unbounded basis functions when two edges attached to the same vertex become collinear.

Remark 13.3. Stable bases were constructed in [14] for the superspline space $\mathcal{S}_{d}^{r, \mu}(\triangle)$, and in [25] for a certain special subspace $\mathcal{S}$ of $\mathcal{S}_{d}^{r, \mu}(\triangle)$, as a first step in constructing quasi-interpolation operators with optimal approximation order. Note that these constructions differ from our algorithm for $\mathcal{S}_{d}^{r, \mu}(\triangle)$ described in Section 11. Compared to the construction in [14], our basis splines have substantially smaller supports in general (see also Remark 13.9). For the space $\mathcal{S S}$ the algorithm in [25] produces basis splines with similar small supports, but does not appear to extend to the full spaces $\mathcal{S}_{d}^{r}(\triangle)$ and $\mathcal{S}_{d}^{r, \mu}(\triangle)$.

Remark 13.4. Well-known finite element results, see e.g. [31], imply that the classical superspline subspaces of $\mathcal{S}_{d}^{1}(\triangle)$ have stable local bases. In [18] we have recently extended this construction to the full spline spaces $\mathcal{S}_{d}^{1}(\triangle)$ with $d \geq 5$. The construction there uses nodal functionals (point evaluation of certain derivatives). Here we have used the linear functionals $\lambda_{\xi}$ which pick off the coefficients of the Bernstein-Bézier form. 
Remark 13.5. No constructions of stable bases for spline spaces with $d<3 r+2$ are known for general triangulations. It is also known [9] that for $d<3 r+2$, there are triangulations for which $\mathcal{S}_{d}^{r}(\triangle)$ has no star-supported basis. However, it is possible to construct stable star-supported bases for some values of $d<3 r+2$ for classes of splines defined on special triangulations using macro-element techniques. These include Clough-Tocher and Powell-Sabin refinements, for example. See $[10,11,26,27]$.

Remark 13.6. For multiresolution applications, it is important to work with sequences of triangulations which are nested. In such cases, the corresponding spline spaces $\mathcal{S}_{d}^{r}(\triangle)$ are also nested, but in general the various superspline subspaces are not. (See the discussion of this "super-spline effect" in $[15,28]$.)

Remark 13.7. Following the proof of Theorem 5.3 (see also the proof of Theorem 9.2 in [25]), it is not hard to establish

Theorem 13.8. Suppose $\mathcal{M}$ is a stable local $M D S$ for a spline space $\mathcal{S}$, and let $\left\{B_{\xi}\right\}_{\xi \in \mathcal{M}}$ be the corresponding dual basis splines. Given $1 \leq p<\infty$, let $B_{\xi, p}:=$ $A_{\xi}^{-1 / p} B_{\xi}$, where $A_{\xi}$ denotes the area of the support of $B_{\xi}$. Then $\left\{B_{\xi, p}\right\}_{\xi \in \mathcal{M}}$ is an $L_{p}$-stable basis for $\mathcal{S}$, i.e., there exist constants $K_{1}$ and $K_{2}$ depending only on $d$ and $\theta_{\triangle}$ such that

$$
K_{1}\|c\|_{p} \leq\left\|\sum_{\xi \in \mathcal{M}} c_{\xi} B_{\xi, p}\right\|_{p, \Omega} \leq K_{2}\|c\|_{p}
$$

for all choices of the coefficient vector $c=\left(c_{\xi}\right)_{\xi \in \mathcal{M}}$.

Remark 13.9. Our construction guarantees that the supports of the basis splines are at $\operatorname{most} \operatorname{star}^{3}(v)$ for some vertex $v$ in general. In some cases the supports can be made smaller. An explicit construction of stable star-supported bases for $C^{1}$ splines can be found in [18]. A careful examination of the construction here shows that for $r=2$, we also get star-supported stable bases. Moreover, the same holds for general $r>2$ provided $d \geq 3 r+\lfloor(r+1) / 2\rfloor+1$, since in this case $D_{2 r}(u) \cap D_{\mu}(w)=\emptyset$ for any two vertices $u, w$ connected by an edge. For $d=3 r+\lfloor(r+1) / 2\rfloor$, it can be shown that our construction leads to $\operatorname{star}^{2}$-supported stable bases. Consequently, star $^{3}$-supported bases only appear for $r \geq 5$.

Remark 13.10. The proofs of Theorems 6.2 and 10.2 are based on Cramer's rule. This idea of choosing a submatrix with the greatest determinant was used already in $[14]$.

Remark 13.11. As in [25], the stable local bases constructed here can be used to build quasi-interpolation operators with optimal approximation order.

Remark 13.12. The fact that stability and local linear independence are mutually exclusive for spline spaces $\mathcal{S}_{d}^{r}(\triangle)$ with $r \geq 1$ was first established for $r=1$ in [18]. The proof was based on nodal determining sets. Here we have used the BernsteinBézier form to establish the same result for general $r \geq 1$. 
Remark 13.13. Our construction of a stable local basis can be easily adapted to the spaces of splines and supersplines on a triangulation on the sphere or a sphere-like surface introduced in [2], see also [3,4]. Indeed, since we are using exclusively Bernstein-Bézier techniques, our construction and the entire argument can be carried over in the same way as was done in [3] for the standard local bases of $\mathcal{S}_{d}^{r, \rho}(\triangle)$.

\section{References}

1. Alfeld, P., Private communication, 1995.

2. Alfeld, P., M. Neamtu, and L. L. Schumaker, Bernstein-Bézier polynomials on spheres and sphere-like surfaces, Comput. Aided Geom. Design 13 (1996), $333-349$.

3. Alfeld, P., M. Neamtu, and L. L. Schumaker, Dimension and local bases of homogeneous spline spaces, SIAM J. Math. Anal. 27(5) (1996), 1482-1501.

4. Alfeld, P., M. Neamtu, and L. L. Schumaker, Fitting scattered data on spherelike surfaces using spherical splines, J. Comput. Appl. Math. 73 (1996), 5-43.

5. Alfeld, P. and L. L. Schumaker, The dimension of bivariate spline spaces of smoothness $r$ for degree $d \geq 4 r+1$, Constr. Approx. 3 (1987), 189-197.

6. Alfeld, P., B. Piper, and L. L. Schumaker, Minimally supported bases for spaces of bivariate piecewise polynomials of smoothness $r$ and degree $d \geq 4 r+1$, Comput. Aided Geom. Design 4 (1987), 105-123.

7. Alfeld, P., B. Piper, and L. L. Schumaker, An explicit basis for $C^{1}$ quartic bivariate splines, SIAM J. Numer. Anal. 24 (1987), 891-911.

8. Alfeld, P. and L. L. Schumaker, On the dimension of bivariate splines spaces of smoothness $r$ and degree $d=3 r+1$, Numer. Math. 57 (1990), 651-661.

9. Alfeld, P. and L. L. Schumaker, Non-existence of star-supported spline bases, SIAM J. Numer. Anal. 31 (2000), 1482-1501.

10. Alfeld, P. and L. L. Schumaker, Smooth finite elements based on Clough-Tocher triangle splits, Numer. Math., submitted.

11. Alfeld, P. and L. L. Schumaker, Smooth finite elements based on Powell-Sabin triangle splits, manuscript, 2000.

12. Boor, C. de, B-form basics, in Geometric Modeling: Algorithms and New Trends, G. E. Farin (ed), SIAM Publications, Philadelphia, 1987, 131-148.

13. Carnicer, J. M. and J. M. Peña, Least supported bases and local linear independence, Numer. Math. 67 (1994), 289-301.

14. Chui, C. K., D. Hong, and R.-Q. Jia, Stability of optimal order approximation by bivariate splines over arbitrary triangulations, Trans. Amer. Math. Soc. 347 (1995), 3301-3318.

15. Dahmen, W., P. Oswald, and X.-Q. Shi, $C^{1}$-hierarchical bases, J. Comput. Appl. Math. 51 (1994), 37-56. 
16. Davydov, O., Locally linearly independent basis for $C^{1}$ bivariate splines, in Mathematical Methods for Curves and Surfaces II, Morten Dæhlen, Tom Lyche, Larry L. Schumaker (eds), Vanderbilt University Press, Nashville \& London, 1998, 71-78.

17. Davydov, O., G. Nürnberger, and F. Zeilfelder, Bivariate spline interpolation with optimal approximation order, Constr. Approx., to appear.

18. Davydov, O. and L. L. Schumaker, Stable local nodal bases for $C^{1}$ bivariate polynomial splines, in Curve and Surface Fitting: Saint-Malo 99, A. Cohen, C. Rabut, and L. L. Schumaker (eds), Vanderbilt University Press, Nashville TN, 2000, 171-180.

19. Davydov, O. and L. L. Schumaker, Locally linearly independent bases for bivariate polynomial splines, Advances in Comp. Math., to appear.

20. Davydov, O., M. Sommer, and H. Strauss, Locally linearly independent systems and almost interpolation, in Multivariate Approximation and Splines, ISNM 125, G. Nürnberger, J. W. Schmidt and G. Walz (eds), Birkhäuser, Basel, 1997, $59-72$.

21. Davydov, O., M. Sommer, and H. Strauss, On almost interpolation and locally linearly independent bases, East J. Approx. 5 (1999), 67-88.

22. Farin, G., Triangular Bernstein-Bézier patches, Comput. Aided Geom. Design 3 (1986), 83-127.

23. Hong, D., Spaces of bivariate spline functions over triangulation, Approx. Theory Appl. 7 (1991), 56-75.

24. Ibrahim, A. and L. L. Schumaker, Super spline spaces of smoothness $r$ and degree $d \geq 3 r+2$, Constr. Approx. 7 (1991), 401-423.

25. Lai, M. J. and L. L. Schumaker, On the approximation power of bivariate splines, Advances in Comp. Math. 9 (1998), 251-279.

26. Lai, M.-J. and L. L. Schumaker, Macro-elements and stable local bases for spaces of splines on Clough-Tocher triangulations, Advances in Comp. Math., to appear.

27. Lai, M.-J. and L. L. Schumaker, Macro-elements and stable local bases for splines on Powell-Sabin triangulations, manuscript, 1999.

28. Le Méhauté, A., Nested sequences of triangular finite element spaces, in Multivariate Approximation: Recent Trends and Results, W. Haussman, K. Jetter and M. Reimer (eds), Akademie-Verlag, 1997, 133-145.

29. Schumaker, L. L., Spline Functions: Basic Theory, Wiley-Interscience, New York, 1981.

30. Schumaker, L. L., Dual bases for spline spaces on cells, Comput. Aided Geom. Design 5 (1988), 277-284.

31. Schumaker, L. L., On super splines and finite elements, SIAM J. Numer. Anal. 26 (1989), 997-1005. 